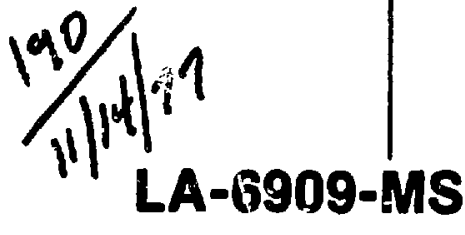

Informal Report

UC-20

Issued: October 1977

\title{
Experiments with Vacuum Interrupters Used for Large DC-Current Interruption
}

\author{
R. W. Warren*
}

"Industrial Staff Mernber. Westinghouse Research Laboratory, Beulah Road, Churchill Boro, Pittsburgh, PA 15235.

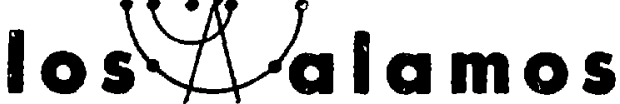

\section{scientific laborafory}

of the University of California

los ALAMOS. NEW MEXICO B754S

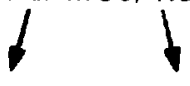

An Affirmative Action/Equal Opportunity Employer 


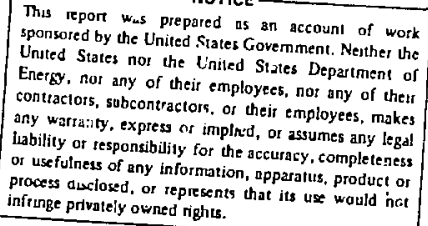

\title{
EXPERIMENTS WITH VACUUM INTERRUPTERS USED FOR LARGE DC-CURRENT INTERRUPTION
}

by

\author{
R. W. Warren
}

\begin{abstract}
Vacuum interrupters have been tested in circuits similar to those used in theta-pinch and tokamak fusion devices. The effects on performance of auxiliary circuit components and axial magnetic fields have been determined, and limits to lifetime caused by mechanical and electrical wear have been measured. Results show that the upper reliable limit of interruption is independent of the auxiliary components but quite dependent on interrupter size and on the axial field.
\end{abstract}

\section{SUMMARY}

More than a dozen vacuum interrupters have been examined to determine their ability to interrupt large dc currents and to successfully sustain the resulting large voltage transients. The importance of auxiliary circuit components such as saturable reactora and R-C "snubber" elements has been assessed as has the effect of varying the electrode gap, its opening speed, and other system variables. The following general rules have been formulated governing $P$, the probability of a successful interruption of a current, $I_{0}$, and $I_{c p}$, the counterpulse current used to create an artificial current zero at which interruption may occur.

(1) $P$ does not depend strongly on $I_{c p}$ if $I_{c p}$ is large enough to achieve a current zero and if the saturable reactor is also large enough.

(2) $P$ does not depend strongly upon $I_{0}$ until the arc voltage becomes noisy. $P$ the $e_{\bar{\imath}}$ drops rapidly with further increases in $\mathrm{I}_{\text {o }}$.

(3) $I_{m}$, the upper limit to $I_{0}$, varies roughly linearly with the diameter of the arcing electrodes.
(4) $I_{m}$ is independent of circuit elements and operational parameters such as saturable reactor, snubber circuit, and opening speed and gap, over a wide range.

(5) There is no significant variation of $I_{m}$ among the vacuum interrupters produced by different manufacturers.

Table I gives $I_{m}$, the upper limit to the interruptible current for all of the interrupters measured at a reliability of 50 and $90 \%$. The last two columns of Table I show the large increase in $I_{m}$ and the decrease in arc voltage which oscurred for those interrupters operated in a strong axial magnetic field. This improvement is substantial and its documentation an important result of this report.

\section{INTRODUCTION}

Many approaches to fusion require storage and rapid transfer of large amounts of energy, posing problems not only for the storage systems but for the transfer circuitry. Power levels are extremely high 


\section{TABLE I}

\section{VACUUM INTERRUPTER CURRENT INTERRUPTION RELIABILITY}

\begin{tabular}{|c|c|c|c|c|c|c|}
\hline \multirow{3}{*}{ Interrupter } & \multirow{3}{*}{$\begin{array}{c}\text { Nominal } \\
\text { Diam } \\
\text { (in.) } \\
\end{array}$} & \multirow{3}{*}{$\begin{array}{l}\text { Electrode } \\
\text { Diami } \\
\text { (cm) } \\
\end{array}$} & \multirow{2}{*}{\multicolumn{2}{|c|}{$\begin{array}{c}\text { Maximum Current } \\
\text { (Im, kA) } \\
\text { Reliability }\end{array}$}} & \multicolumn{2}{|c|}{ Axial Fiold } \\
\hline & & & & & (\% Increase & (\% Decreabe in \\
\hline & & & 50\% & $\underline{90 \%}$ & & \\
\hline A1 & 4 & 2 & 6. & 5 & & \\
\hline A2 & 4 & 2 & 8 & 7 & 10 & 15 \\
\hline A3 & 4 & 5 & 13 & 12 & 25 & 50 \\
\hline B1 & 4 & & 6 & 5 & & \\
\hline A4 & 5 & 7 & 17 & 14 & & \\
\hline A5 & 5 & 7 & 18 & 15 & 70 & 10 \\
\hline A6 & 5 & 8 & 16 & 14 & & \\
\hline A7 & 5 & 9 & 19 & 17 & 80 & 10 \\
\hline A8 & 7 & 10 & 24 & 22 & & \\
\hline A9 & 7 & 12 & 26 & 24 & & \\
\hline Prototype 1 & 7 & 10 & $>37$ & $>37$ & a & \\
\hline Protritype 2 & 7 & 13 & 15 & 13 & $>100$ & 50 \\
\hline $\mathrm{Cl}$ & 8 & 11 & 28 & 26 & & \\
\hline
\end{tabular}

-Interrupter has an internal axial field coil.

${ }^{\circ} \mathrm{A}, \mathrm{B}$, and $\mathrm{C}$ refer to three different manufacturers.

because the energies involved in reactor-size devices are typically $\sim 10^{7} \mathrm{~J}$ and tranefer times range from 1 to $10^{-5} \mathrm{~s}$. A major concern is the switching required in these systems, particularly any interrupting switch. The use of solid state thyristor switches or "plasma valves" of various kinds is attractive because of their anticipated long life. However, they are prohibitively expensive when compared with the mechanical switches which are now used. But mechanical switches cannot achieve the goal of millions of operations required for reactor applications. Because of this cost-performances spiit, other switching components and concepts which may be inexpensive and may provide a long operating life should be examined. This report describes the beginning of such an examination of vacuum intezrupters.

During the last several years the Los Alamos Scientific Laboratory (LASL) has been pursuing research on current interruption with commercial vacuum interrupters for "se in an inductive energy storage and transfer system for the Scyllac Fusion
Test Reactor (SFTR).' More recently, development has been applied to the Tokamak Ohmic Heating (TOH) circuitry. To meet the requirements of SFTR, i.e., $500 \mathrm{MJ}$ energy storage to be delivered to the coil in $1 \mathrm{mc}$, a system of modules was developed. The maximum current and voltage for these modules were set rather arbitrarily at $26 \mathrm{kA}$ and 60 kV, which were felt to be the highest current and voltage that the various components could easily sustain. These limits and the transfer time of $1 \mathrm{~ms}$ determined the $400-\mathrm{kJ}$ module size. A diagram of the circuit of one module is shown in Fig. 1A, where $\mathrm{L}_{\boldsymbol{b}}$ is the load coil, $\mathrm{L}_{\boldsymbol{b}}$ is the superconducting coil, and HP is a generator which raises the current in $\mathrm{L}_{\text {}}$ and switch $S_{1}$ to $26 \mathrm{kA}$ in several minutes. At this time $S_{1}$ is opened and the current is transferred to the circuit containing $L_{\infty}$ and $C_{9}$. If $L_{0}$ and $C_{3}$ have the correct values, all of the energy originally stored in $\mathrm{L}_{\mathbf{b}}$ is transferred to $\mathrm{L}_{\mathbf{b}}$ in $1 \mathrm{~ms}$. If $\mathrm{S}_{\mathbf{s}}$ is closed when this transfer has been accomplished, the energy can be held in $L_{s o}$ for a time given by its $L / R$ decay constant. 
One of the difficulties with this circuit is the need for 8 switch $S_{1}$ which can successfully interrupt a 26$\mathrm{kA}$ current and withstand a $60-\mathrm{kV}$ recovery voltage. Vacuum interrupters were selected as probable candidates for $S_{1}$, but because they were developed for ac uses, little was known about their performance in this unusual application. Accordingly, a special apparatus was set up to test the interrupters and their possible auxiliary components.

This report discusses the interrupter circuit, functions of its components, and progress made on this test program. An important feature of this program has been a gradual broadening to include interrupter tests for other applications. Tokamaks, in particular, need interrupters for their ohmic heating systems with ratings as large or larger than those of SFTR.

\section{PROGRAM PLAN}

The primary purpose of the experiments is to investigate the performance of standard vacuum interru, ters. Performance is evaluated by examining (1) the reliability of interruption vs current, (2) the operating life of an interrupter, and (3) the dependence of these properties on the variables under our control.

These variables include the following important parameters:

(1) the magnitude of the counterpulse current,

(2) the type of interrupter, i.e., size and manufacturer,

(3) how the interrupter is operated, i.e., opening speed and electrode spacing,

(4) temperature,

(5) the presence of magnetic fields,

(6) the properties of the saturable reactor component, and

(7) the properties of the snubber component.

Because the measurements are quite timeconsuming, an orderly testing approach is required to arrive at useful results in the shortest time. The following plan was used.

1. Choose a "standard" interrupter and operate it under the simplest circuit conditions, that is, no snubber, no saturable reactor, and the simplest environmental conditions (room temperature and no magnetic field). Choose a standard electrode opening speed and gap.
2. Vary $I_{x p}$, the counterpulse current, over a wide range and determine $P\left(I_{c p}\right)$, the probability of interruption.

3. Vary $L_{0}$, the interrupter current, and repeat step 2, that is, establish $P\left(I_{0}, I_{c p}\right)$ under standard conditions.

4. Vary the moving electrode's speed and gap at counterpulse and determine their effects on $P\left(I_{0}, I_{c p}\right)$ over a limited range of $I_{0}$ and $I_{c p}$ where $P$ is near $50 \%$ and thus particularly sensitive to change.

5. Install the snubber circuit and saturable reactor separately and test their effects on $P\left(L_{0}, I_{c p}\right)$ over a limited range of $L_{0}$ and $I_{c p}$ and for the new standard speed and gap determined to be optimum in step 4.

6. Test samples of all commercially available interrupters over $a$ wide range of $I_{0}$ and $I_{c p}$ and for the standard speed and gap and for an optimum standard saturable reactor and snubber circuit determined in step 5 .

7. Investigate effects of different electrode temperatures on $\mathbf{P}$ for the standard interrupter under standard conditions.

8. Investigate effects of magnetic fields on $P$.

9. If necessary, investigate interrupter performance under combinations of nonstandard canditions.

10. Evaluate the lifetime of the standard interrupter under standard conditions.

The following sections go into considerable detail in discussing our results for each part of this plan. These sections are organized to follow the order of the plan.

\section{CIRCUIT DESCRIPTION}

A full diagram of the test circuit is shown in Fig. 2, which differs from Fig. 1A for four reasons. First, the basic operating mode is somewhat different. In Fig. 1A, the energy originally present in $\mathrm{L}_{\text {s }}$ is transferred in part to $C_{2}$ and then to $L_{0}$ where it is trapped by reclosing switch $S_{1}$. In Fig. $1 B$, which is a modified form of Fig. 1A, the energy originally in $L_{s}$ can be transferred to $C_{2}$ and then back into $L_{4}$ where it is trapped by closing switch $S_{2}$. This circuit modification eliminates one major component, $L_{e}$, but does not change the requirements on $S_{1}$, the interrupter. The test circuit includes this modification. 


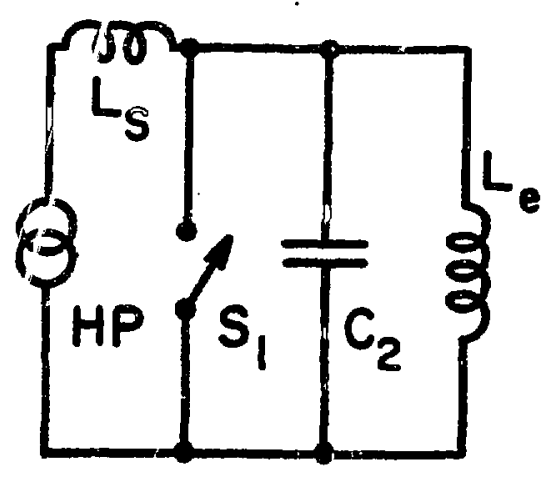

A

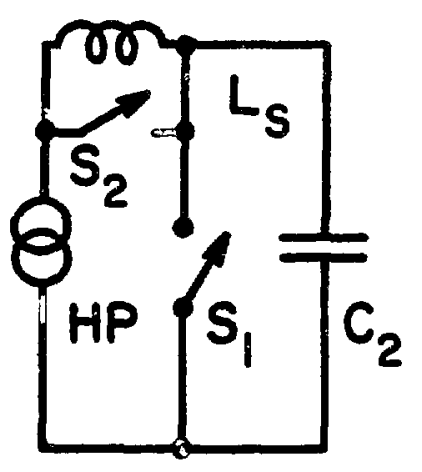

B

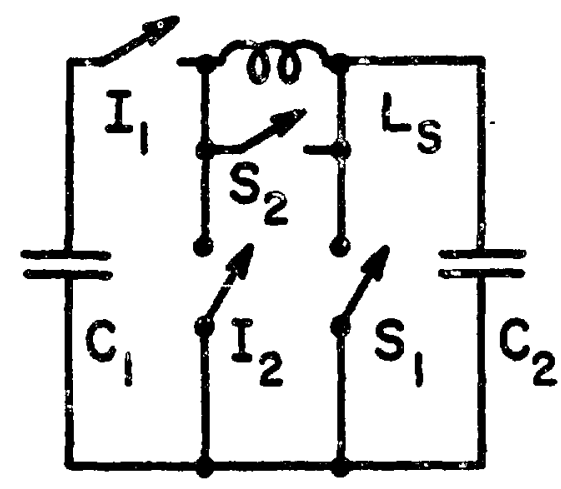

C

Fig. 1.

Progressive modification of energy transfer modules.

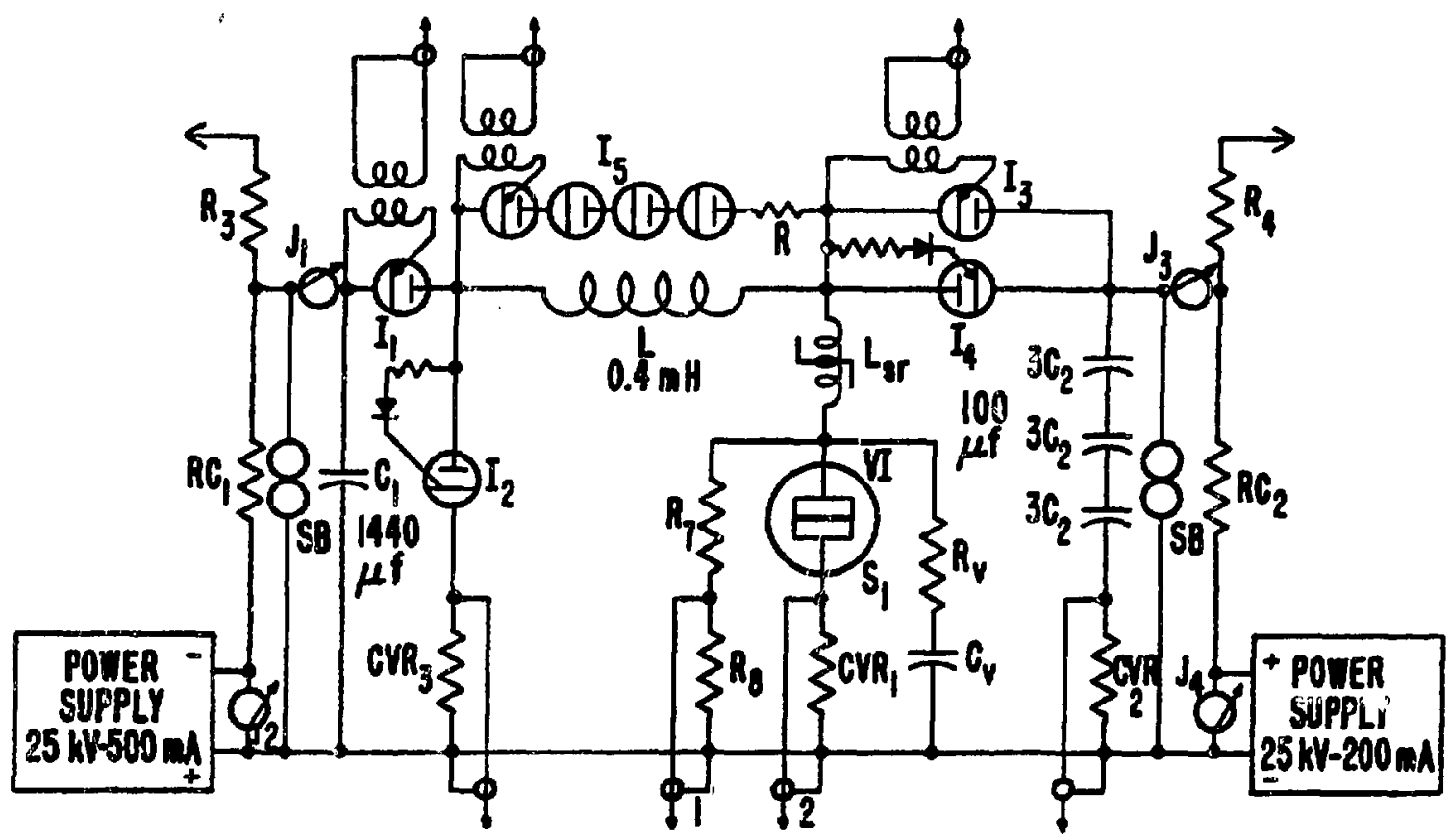

Fig. 2.

Test circuit.

Second, in Figs. 1A and 1B, a dc generator was used to pump energy into $L_{\text {n }}$ over a time of several minutes. This was made possible by the superconducting nature of $\mathrm{L}_{3}$. Figure $1 \mathrm{C}$ shows a further modification of Figs. $I A$ and 1B; energy is delivered to nonsuperconducting $L$. from the capacitive energy storage aubsystem composed of capacitor $C_{1}$ and ignitrons $I_{2}$ and $I_{2}$, instead of from HP. Figure
1C is a simplified form of the real test circuit shown in Fig. 2. In the circuit shown in Fig. 2, $C_{1}$ is charged through switch $J_{1}$ over several minutes by the $25-\mathrm{kV}$ power supply; ignitron $I_{1}$ is fired, initiating an LC uscillation with a 4 -ms period involving the components $C_{k}, L_{2}, L_{n r}$ and switch $S_{1}$. After a quarter period, $C_{1}$ is discharged and the current in $L_{0}$ reaches its maximum. $I_{2}$ then fires automatically so 
that the current in $\mathrm{L}_{0}$ decays exponentially through $I_{2}$ and $S_{1}$ with a time constant of $\sim 100 \mathrm{~ms}$. This 100 ms interval is adequate to allow completion of our experiments that concentrate on the opening of switch $S_{1}$ and its successful interruption of the current.

The third difference between Figs. 1A and 2 is the extra equipment shown in Fig. 2 which assists in the interruption or serves some other purpose. The 15$\mathrm{kV}$ supply and ignitrons $\mathrm{I}_{3}$ and $\mathrm{I}_{4}$, for example, allow $\mathrm{C}_{2}$ to be charged belore the interruption is to be accomplished. At the time of interruption, $I_{s}$ is triggered, generating a counterpulse current $I_{c p}$ through $S_{1}$ of the opposite sign from $I_{o}$, the current being interrupted. If the magnitude of $I_{c p}$ and $I_{0}$ are approximately equal, the net current through $S_{1}$ is very small and is easy to interrupt. $L_{a r}$ is a saturable reactor which helps to hold the net current near zero for long times. $R_{v}$ and $C_{v}$ are parts of the snubber circuit which also helps to extinguish the arc that forms when $S_{1}$ opens. Ignitron $I_{s}$ and resistor $R$ act as the final "dump" for the energy originally in $C_{1}$. $R_{C_{1}}$ and $J_{2}$, as well as $R_{C_{2}}$ and $J_{4}$, provide a means of removing energy from capacitor banks $C_{1}$ and $C_{2}$ in an emergency. The shorting balls, $\mathrm{SB}_{1}$ and $\mathbf{S B}_{2}$, provide aseurance that $C_{1}$ and $C_{2}$ are uncharged at the end of an experiment.

The fourth difference between Figs. 2 and $A$ is the provision made in Fig. 2 for measurement of the crucial current and voltage parameters. This in. cludes several low-impedance $(1 / 4-m \Omega)$, low-

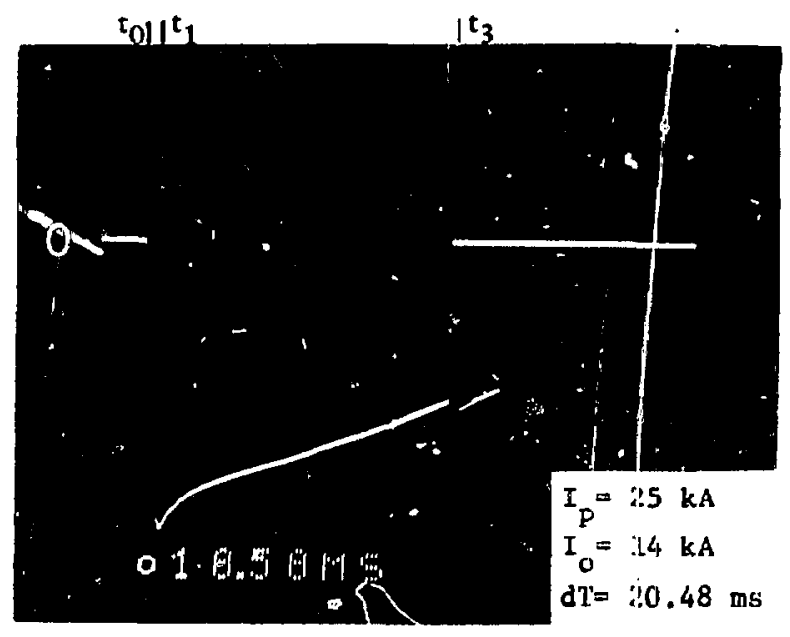

Fig. 3.

Interrupter current us time. inductance shunts (frequency response to $19^{8} \mathrm{~Hz}$ ) which can be placed at various points in the circuit to allow current measurements, and various voltage probes (frequency response to $10^{\circ} \mathrm{Hz}$ ) used similarly. The kinds of signals commonly observed and often presented in this following discussions are shown in Figs. 3-7. Figure 3 shows the current through the interrupter on a long time scale, i.e., 20 $m s$ or so. The quantities in the inset $\left(I_{p}, I_{o}\right.$, and $\left.d T\right)$ are, respectively, the peak current, the current at interruption, and the overall time interval displayed in Fig. 3. Figure 3 shows, at $t_{o}$, the sinusoidal current rise after $I_{1}$ fires; at $t_{1}$, the exponential decay after $I_{2}$ fires; at $t_{3}$, the firing of the counterpulse; and after this, sometimes, the occurrence of restrikes.

Figure 4 shows the voltage across the interrupter on a similar time scale. $V$ in the inset is the overall voltage interval displayed, i.e., 250 volts full scale. On it can be seen at $t_{o}$ the cosinusoidal voltage variation corresponding to the sinusoidal current rise; at $t_{1}$, a transient when $I_{2}$ fires; at $t_{2}$, a step in voltage when the interrupter $S_{1}$ opens, followed by an approximately exponentially increasing voltage, full of noise, which is the arc voltage; and at $t_{3}$, the off-scale energy-transfer voltage transient.

Figure 5 shows the current near $t_{3}$, counterpulse time, on an expanded time scale of $100 \mu \mathrm{s}$ or so. On it can be seen at $t_{a \mathrm{a}}$ the initiation of the counterpulse, and at $t_{3 b}$, the first current zero. Sometimes a second current zero occurs as seen at $t_{3 c}$ on Fig. 5. Figure 6 shows a vertical expansion of Fig. 5. Figure 7 shows

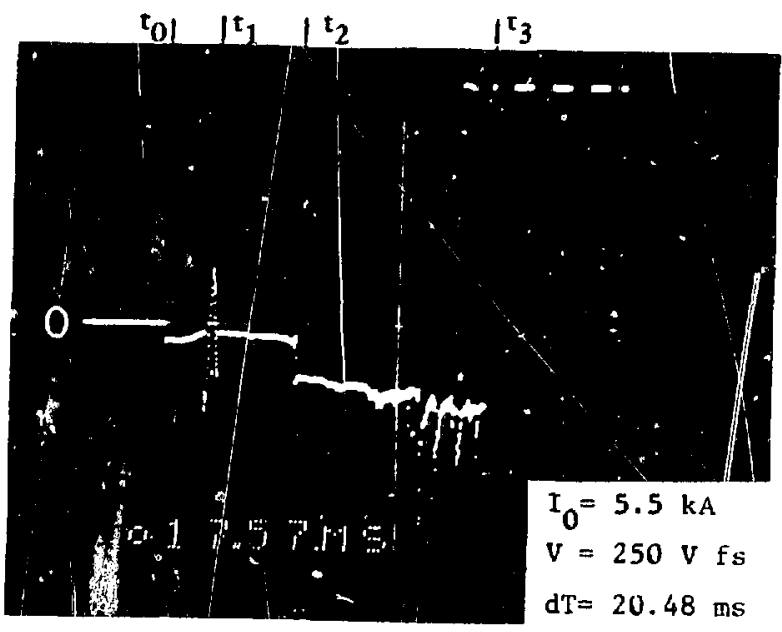

Fig. 4.

Interrupter voltage us time. 


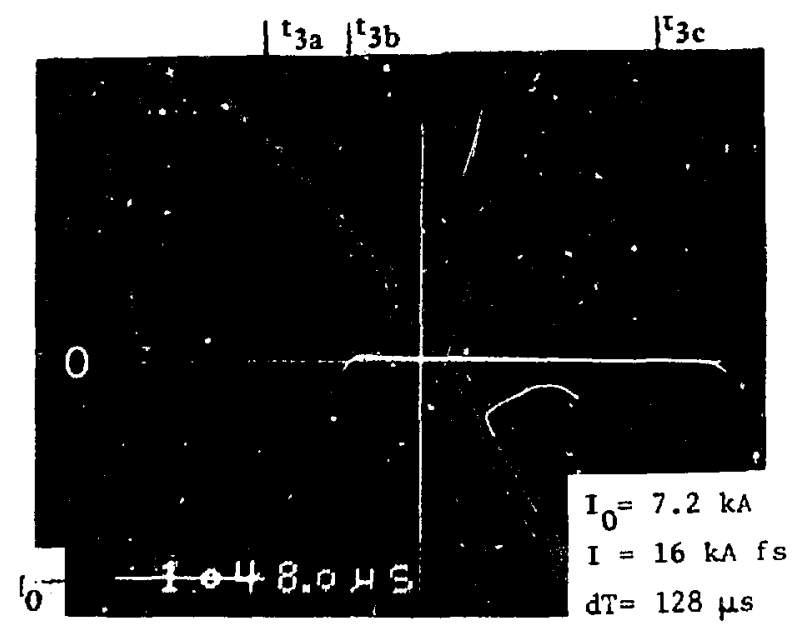

Fig. 5.

Current near counter-pulse time.

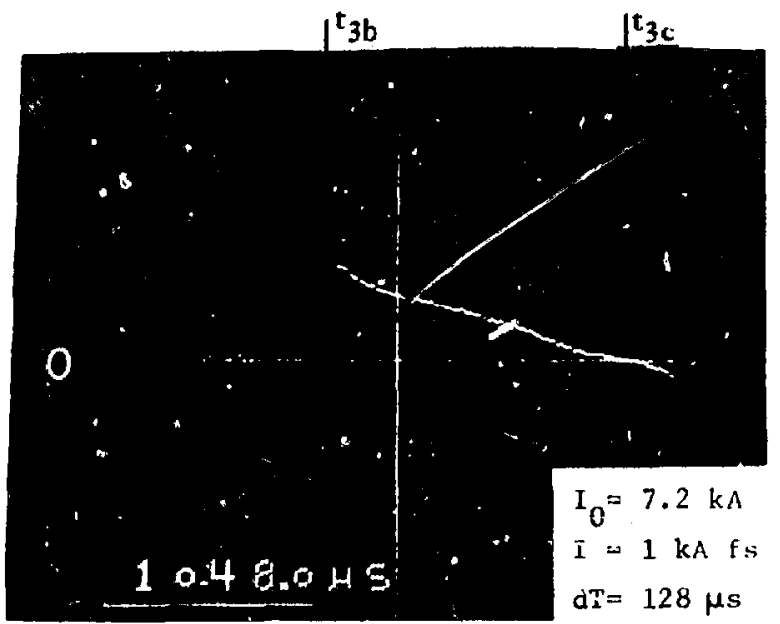

Fig. 6.

Expanded trace of Fig. 5.

the interrupter voltage near $t_{s}$ on a similarly expanded time scale. On it can be seen at $t_{9 a}$ the voltage jump caused by the counterpulse current; at $t_{3 k}, a$ drop in voltage caused by the first current zero; and at $t_{3 a}$, a sudden drop in voltage caused hy the end of the counterpulse. The voltage then begins a sinusoidal increase as the energy is transferced into $\mathrm{C}_{2}$ with a time constant of $\sim 1 \mathrm{~ms}$.

To keep all of these times straight, a list, of thern is presented here:

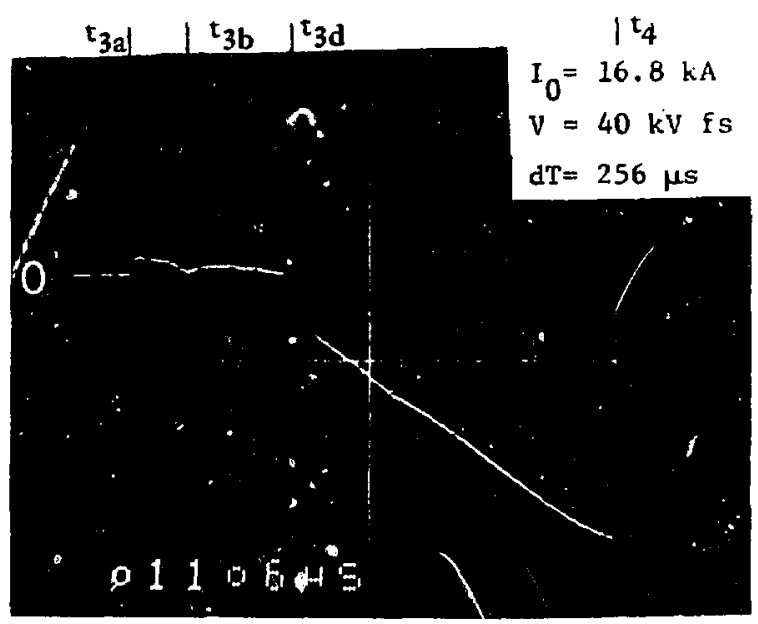

Fig. 7.

Voltage near counter-pulse time.

$t_{0} \quad I_{1}$ fires, current flows out of $C_{1}$

$t_{1} \quad I_{2}$ fires, beginning of exponential decay of current

$t_{2} \quad$ iriterrupter opens, arc voltage rises rapidly

$\mathrm{t}_{3} \quad \mathrm{I}_{8}$ fires, counterpulse started

$t_{s a} \quad I_{s}$ fires, initiation of counterpulse

$t_{\mathrm{ab}} \quad$ first current zero

$t_{y c} \quad$ second current zero

$t_{s d} \quad$ end of counterpulse.

\section{FXPERIMENTAL PROCEDURE}

\section{A. The Standard Interrupter Setup}

The largest interrupters made by GE, Westinghouse, and ITT-Jennings are shown in Fig. 8. The family of Westinghouse interrupters, i.e., the 4 -in., 5-in., and 7-in. sizes are shown in Fig. 9. We have chosen as our standard interrupter the most common 7-in. Westinghouse interrupter \#WL23231. A view of one electrode and the inside of this interrupter is shown in Fig. 10. This interrupter is normally mounted on an actuator mechanism which is powered by an electronic driver circuit, all of which can be triggered by an electric pulse to open 


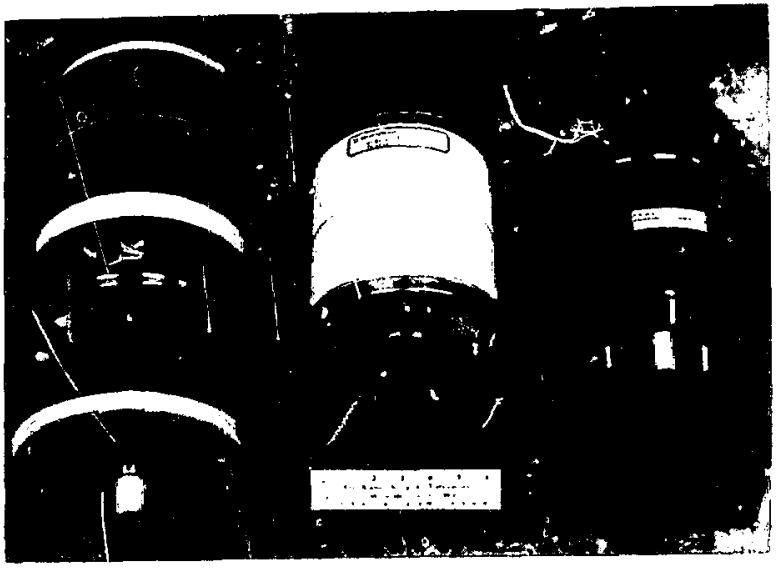

Fig. 8.

Family of largest interrupters, made by General Electric, Westinghouse, and ITT. Jennings (from left to right).

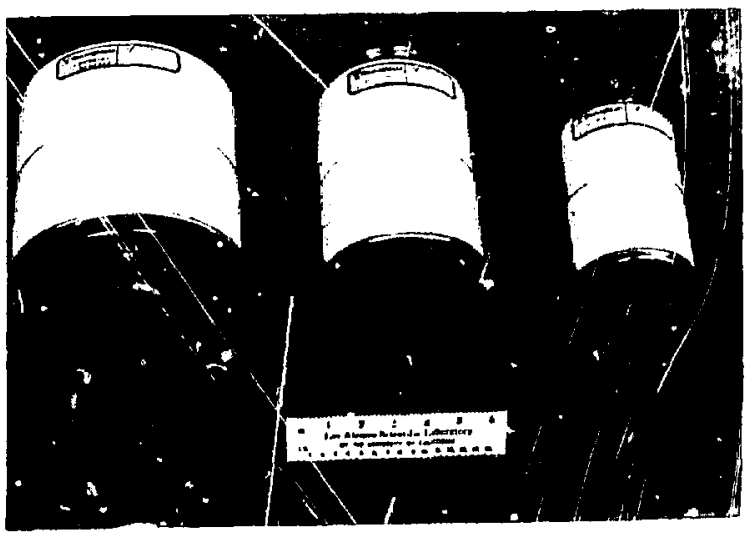

Fig. 9.

Family of different sized interrupters made by Westinghouse.

and a different electrical pulse to close. The actuator and driver are standard components purchased from Ross Engineering Corp.*

Figure 11 shows a 7-in. interrupter mounted on the actuator. The opening speed can be varied to a maximum of $\sim 2.5 \mathrm{~m} / \mathrm{s}$ by changes in the driver circuit. The electrode gap opens to a maximum of $\sim 1.5$ $\mathrm{cm}$. The standard opening speed and gap were chosen to be $2.5 \mathrm{~m} / \mathrm{s}$, and $0.7 \mathrm{~cm}$.

-Ross Engineering Corporation, 559 Westchester Drive, Cambell, CA 95008 .

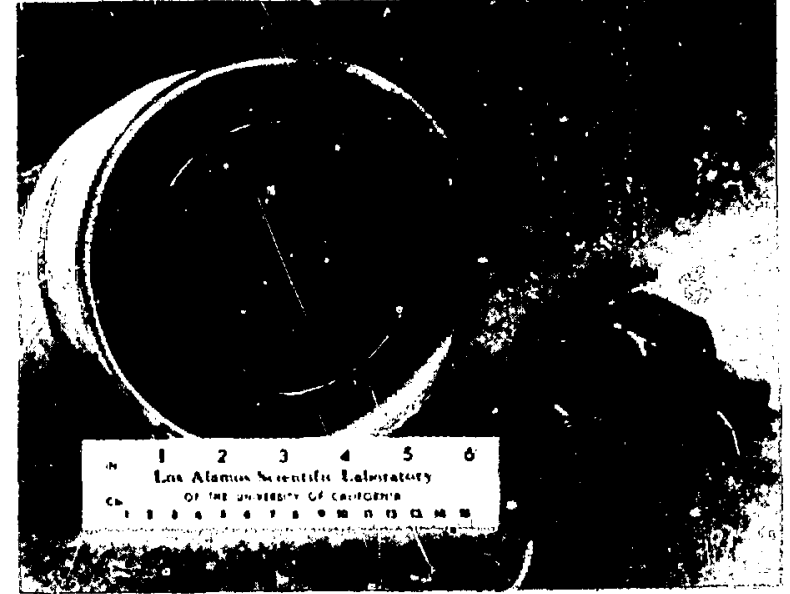

Fig. 10.

Inside of 7-in. inter-'spter.

The opening speed is usually measured by an optical technique. A narrow bean of light is passed from the source to a photocell. The beam is intercepted by a lightweight "comb" molinted on the moving electrode. This comb alternately passes and interrupts the beam and generates electrical pulses in the photocell. Figure 12 shows a scope trace of these pulses, and Fig. 13 shows a resulting plot of electrode displacement is time. The instant of eler.trode separation is simultaneously determined by a simple resistance measurement between the electrodes. Occasionally the electrode motion is measured with a linear potentiometer. This allows a higher spatia! resolution of the motion but slows it down due to the extra mass added to the moving electrode by the potentiometer and its clamp.

When the two capacitor banks have been charged to the desired potential four pulses are delivered; three to the ignitrons $I_{1}, I_{3}$, and $I_{B}$, and one to the driver. First $I_{1}$ is triggered to initiate the current in $L_{a}$ and $S_{1}$; then the driver is triggered to open the interrupter. Ignitron $I_{b}$ is triggered to generate the counterpulse, and finally $I_{\delta}$ is triggered to trap the energy in $L_{s}$ and damp it out in $R$. The delay between these events is $\sim 1 \mathrm{~ms}$ before the interrupter spens, $\sim 3 \mathrm{~ms}$ before the counterpulse is generated, and $1 \mathrm{~ms}$ before the energy is trapped back in $L_{3}$. Figure 3, a slow-speed trace of the current through $S_{1}$, shows two of these events, the initiation of the 


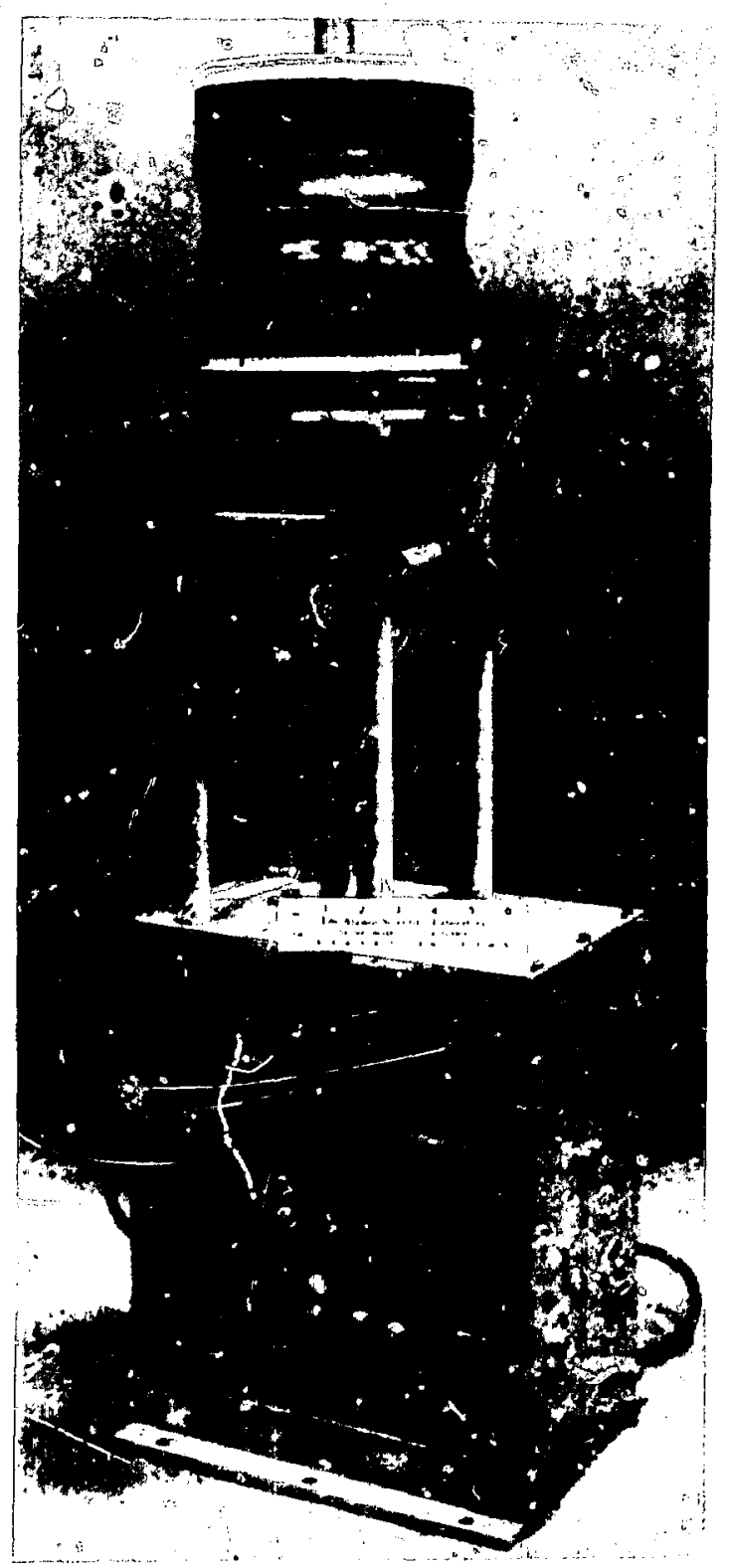

Fig. 11.

Interrupter and its actuator.

current at $\mathfrak{t}_{0}$ and its interruption at $t_{3}$. Figure 4 , a trace of the voltage across the interrupter on the same time scale, shows $t_{2}$, the time when the interrupter opens. Finally, much faster sweeps show details of the counterpulse current and voltage and the interruption phenomenon. Figures 5,6 , and 7 show such traces.

All of the various wiggles and bumps shown in Figs. 3-7 have a significance which should be un-

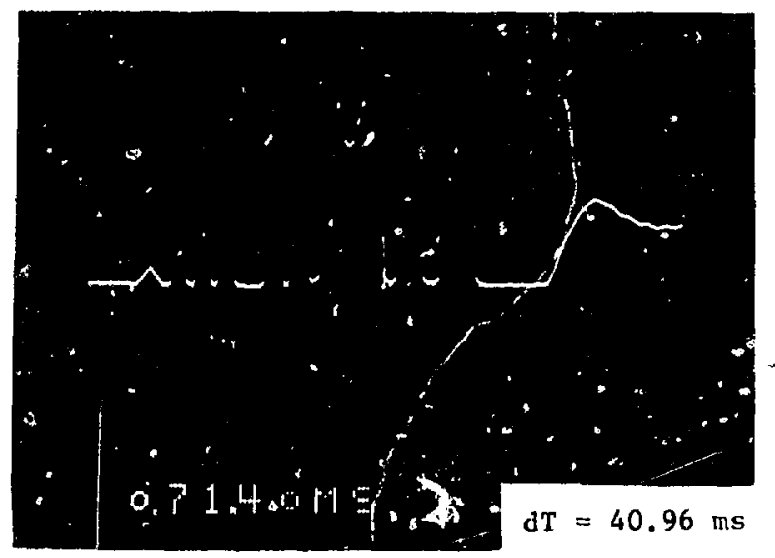

Fig. 12.

Photoelectric record of electrode motion.

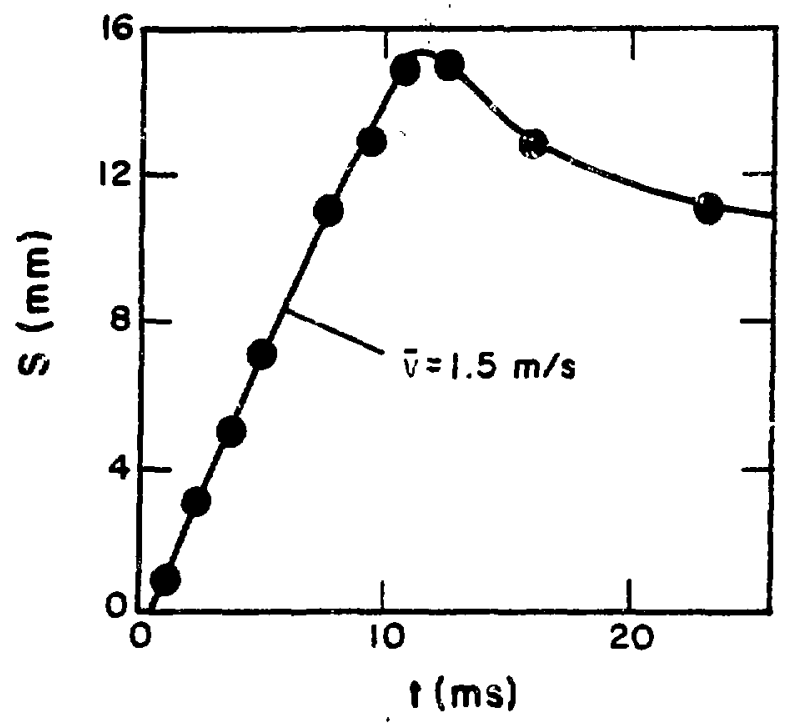

Fig. 13.

Electrode position vs time.

derstood. The sinusoidal current rise of Fig. 3, for example, results from the ringing of the LC circuit composed of $C_{1}$ and $L_{8}$. The exponential decay shown in Fig. 3 is governed by the magnitude of $L_{8}$, its resistance, and the voltage drops across ignitron $I_{2}$ and the arcing interrupter. The overall sinusoidal shape of Fig. 7 is determined by the ringing of the $L C$ circuit composed of $L_{8}$ and $C_{2}$. The cosinusoidal voltage curve at the beginning of Fig. 4 is a result of 
the sinusoidal current shown in Fig. 3 and the inductance of the interrupter and its leads through which this current passes. The jump at time $t_{2}$ is proportional to the isc voltage. It reveals some of the arcing phenomena and differs from interrupter to interrupter and for the same interrupter under different operating conditions. Finally, the details of Figs. 5 and 6 are closely related to circuit and arcing phenomenon. In Fig. 5, for example, the shape of the coul ierpulse current's leading edge is determined by $C_{2}$ and the stray inductance in the circuit. The total current passes through zero twice, once at $t_{3 b}$ and once at $t_{a c}$. Interruptions are commonly observed at both the first and second current zero and are occasionally observed at third and higher zeros. An interruption at either the first or second zero is considered to be auccessful. The only difference from the circuit point of view is a slight delay in the generation of the voltage wave of Fig. 7 in the case of a second zero. hx will be discussed later, this delay becomes even smaller if saturable reactors are used in the circuit.

\section{B. Determination of $\mathbf{P}\left(\mathbf{I}_{\mathrm{cp}}\right)$}

The voltage on $C_{1}$ is adjusted to produce a desired current $I_{0}$ through $S_{1}$ at time $t_{3}$ when the counterpulse is generated. The voltage on $\mathbf{C}_{2}$ is adjusted to produce a desired counterpulse current $I_{c p}$. Delay times $t_{k}, t_{k}$, and $t_{4}$ are adjusted to the standard settings and the apparatus is set into operation. The resulting interruption is monitored on four oscilloscope channels and recorded as successful or not. In general two different kinds of failures are observed. In Type A failures, the arc fails to interr:'pt at all, i.e., the current through $S_{1}$ continues without any significant voltage appearing across it. In Type $B$ failures, the arc current reignites only after a large voltage has appeared across $S_{1} . I_{c p}$ is varied and successful interruptions at the first (Type 1) or second (Type 2) zero are distinguished from Type A or B failures. Figure 14 shows a typical result for a given $\mathrm{I}_{0}$, where $\mathbf{P}$, the probability of interruption, is plotted against $I_{c p}$. If $I_{c p}<I_{o}$, no interruption occurs. We label this region "O." If $\mathrm{I}_{\mathrm{cp}}$ is slightly greater than $\mathrm{I}_{\mathrm{o}}$ interruptions are all successful and at the first zero, i.e., Type 1 . If $I_{c p}$ is further increased, successful Type 2 interruptions become more numerous. Finally, with high enough values of $I_{c D}$, failures of both Types A and B occur. On Fig. 14 we have designated two special points, the values of $I_{c p}$ at which $\mathrm{P}$ is $90 \%$ and $50 \%$. These points will be discussed below.

\section{Determination of $\mathbf{P}\left(\mathbf{I}_{0}, \mathbf{I}_{\mathrm{cp}}\right)$}

The above measurements were repeated at different values of $I_{0}$ until the maximum interruptible current $I_{m}$ was covered. Figure 15 shows the results for standard conditions, where $I_{c p}$ is plotted vs $I_{0}$

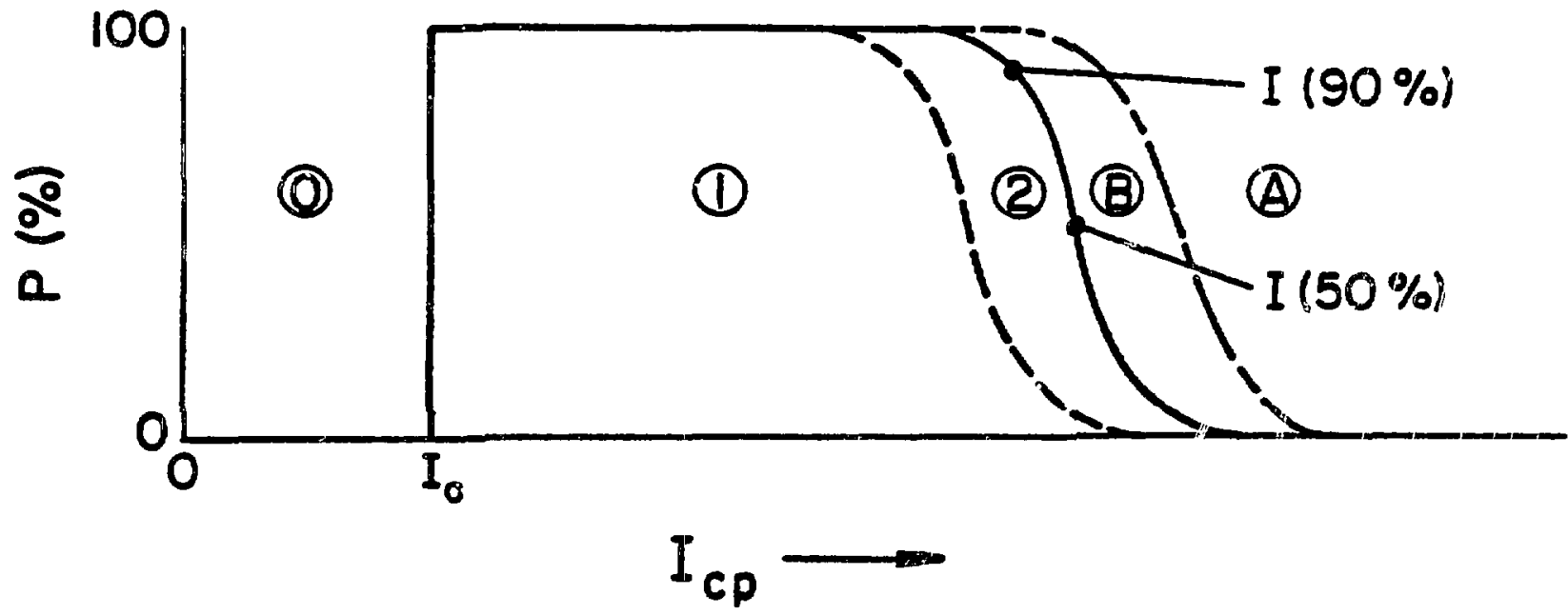

Fig. 14.

Probability of interruption vs counter-pulse amplitude. 


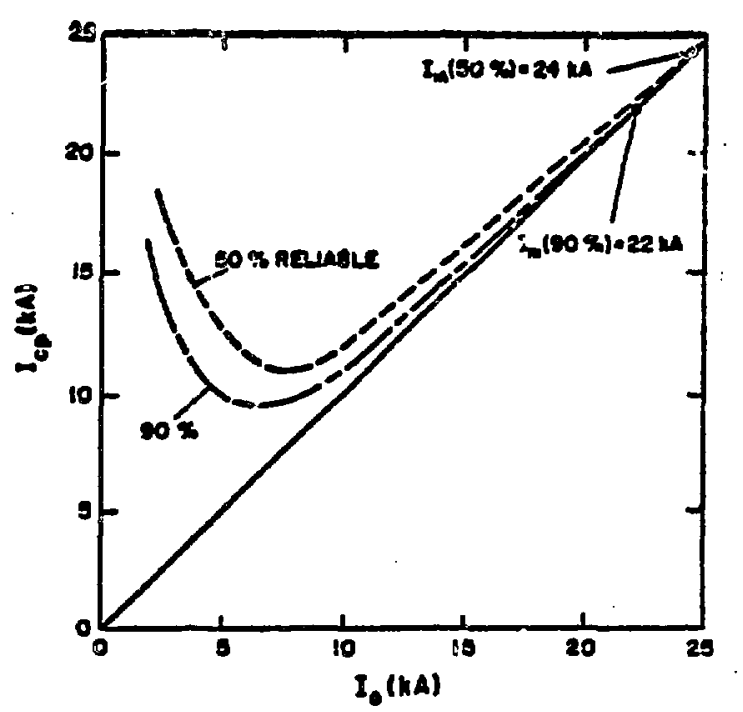

Fig. 15.

Probability of interruption us $I_{0}$ and $I_{\mathrm{cp}}$.

and contours of constant $P$ are shown. The straight line at $4.5^{\circ}$ represents the condition where $I_{c p}=I_{0}$. If $I_{c p}<I_{0}$, no current zeros and, therefore, no interruption occurs. An interesting feature of these curves is that as $I_{0}$ approaches $I_{m}$, the range of $I_{c p}$ that leads to a successful interruption grows steadily smaller. For the interrupter shown in Fig. 15, $I_{m}=24 \mathrm{kA}$ when $\mathrm{P}=50 \%$ and $22 \mathrm{kA}$ when $\mathrm{P}=90 \%$.

\section{Dependence of $\mathbf{P}$ on Opening Speed and Gap}

Adjustments were made in $I_{o}$ and $I_{c p}$ until $I_{0}$ was near its cipper limit $I_{m}$ and $P$ was near $50 \%$. $P$ is then especially sengitive to small variations in any independent variable. The gap was then varied, keeping the opening speed and $I_{0}$ and $I_{c p}$ constant; and $P$ was measured. A dependence of $P$ on gap was found for several different interrupters. A typical example is shown in Fig. 16. As the gap was increased $P$ increased untii a critical gap go was reached. For larger gaps, $P$ fell to a much lower value. This experiment was repeated with an opening speed lower than stundard by a factor of 1.4. Exactly the same dependence on gap was noted. Thus the gap appaars to be a crucial parameter in determining $P$, not arcing time. In Fig. 16 the overall variation in $P$ with gap size is large, but it corresponds to a change in $I_{m}$ with gap size of only $20 \%$ or 80 .

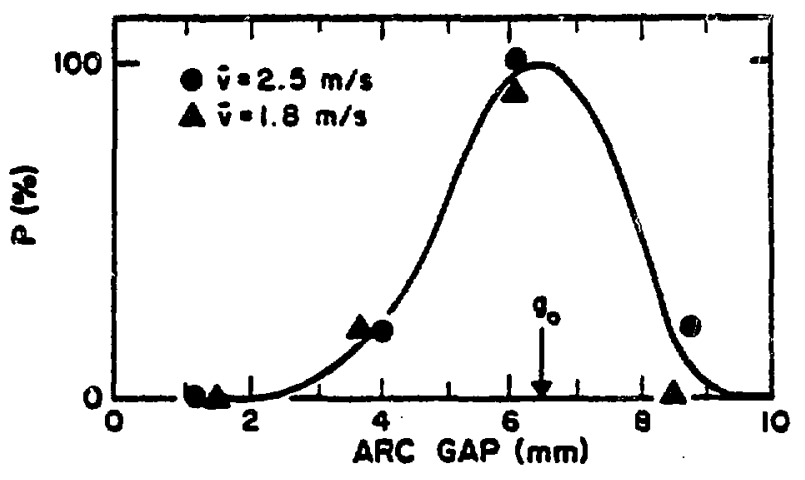

Fig. 26.

Probability of interruption vs arc gap.

P gradually increases as the gap increases because the recovery voltage is applied across larger gap, producing a smaller field and, therefore, a lower restrike probability. We found that as the gap at counterpulse is increased, more and more failures are of Type $B$ and these restrikes occur at higher and higher voltages, i.e., the restrike tends to occur at a criticel field.

The drop in $\mathbf{P}$ when the gap size exceeds go requires a more complex explanation. The voltage across the arcing interrupter was examined and traces like those shown in Fig. 4 were obtained. For short arcing times (st $t$ gaps) the trace is quite smooth. For long times (long gaps) it is very noigy. The noise always starts it a time which corresponds to a gap equal to $\mathrm{Bo}_{0}$. Thus, an important correlation has been established; whenever the gap exceeds $g_{0}$, the voltage becomes noisy and $P$ drops to low values. It is believed that the onset of noise signifies the formation of a constricted arc and an anode hot spot. The hot spot, in turn, assists in the reignition of the arc and, thus, explains the drop in $\mathrm{P}$ when the gap exceeds $g_{0}$.

\section{E. Saturable Reactor}

A saturable reactor minimizes the net current flowing through switch $S_{1}$ for a time ot long enough to allow a significant recombination of the plasma left over from the much higher currents carried. just before the counterpulse. This critical time $\delta \mathrm{t}$ is in the range 10 to $100 \mu s$ for vacuum interrupters. The 
desired value of net current is as low as possible. The standar ${ }^{\prime}$ reactor was chosen as one composed of several laminated gapless cylindrical cores wound from a 4-mil strip of silicon steel. The dimension of each core is 1 -in. i.d. and 4 -in. o.d. by 4 -in. long. The flux rating of one core is $0.008 \mathrm{~Wb}$. The time before a single core becomes saturated, $\epsilon$, is calculated to be $4 \mu \mathrm{s}$ from the relationship $V_{\epsilon}=\phi$, where $V$ is the counterpulse voltage applied to a reactor $(\sim 2 \mathrm{kV})$ and $\phi$ is its flux rating $(0.008 \mathrm{~Wb})$. To achieve the 10 - to $100-\mu \mathrm{s}$ critical time, from $2-25$ cores are necessary. A standard of 10 cores was chosen. These cores are arranged in the circuit by simply threading the insulated lead from $L_{1}$ to $S_{1}$ thirough the stack of reactors once.

This arrangement is shown in Fig. 17, where six such cores are shown on the right side of the picture. The left side shows a stack of split " $C$ " cores with the same flux rating. A secondary vinding is shown threading these $\mathrm{C}$ cores. The use of multiple turns threading the reactors would not extend $\delta$ t but would have some effect on the net current before and during saturation.

A demonstration of the behavior of our standard saturable reactor is shown in Figs. 5, 6, 18, and 19, which show the current through $S_{1} . S_{1}$ is not opened for these tests, thus no interruption occurs. Figure 18 shows the case where $I_{c p}$ is slightly too low to bring the net current to zero. The net current then has a sinusoidal oscillation whose frequency is determined by $\mathrm{C}_{2}$ and the stray inductances. The saturabie reactor is completely saturated at all times contributing no inductance to the circuit. Figure 5 shows a case where $I_{c p}$ is slightly larger. The saturable reactor has unsaturated at $t_{3 n}$ and become saturated again at $t_{3 \mathrm{~d}}$. It holds the net current to a low value for almost $70 \mu \mathrm{s}$. Figure 6 is a vertical expansion of Fig. 5 by a factor of 16 to show the detailed shape of the net current. Finally, Fig. 19 shows what happens when $I_{\mathfrak{r p}}$ is excessively large. In this case the saturated core is driven by the counterpulse through the unsaturated region back into saturation in the opposite direction and then the core traces its path. The intermediate saturated interval generates the "blip" seen in Fig. 19.

The only uncxpected behavior demonstrated by these measurements is the "reversed" slope of the net current shown most clearly in Fig. 6. In an ideal inductor, i.e., one with infinite permeability and no

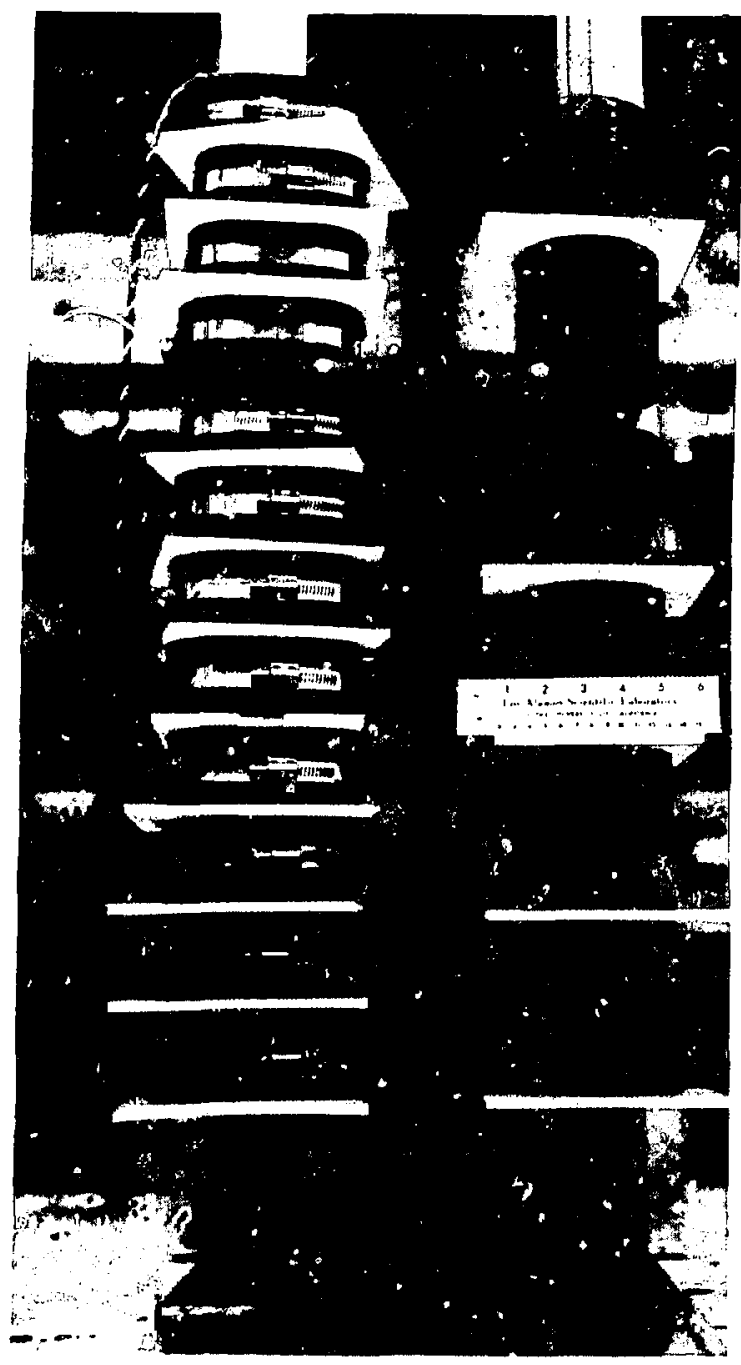

Fig. 17.

Two stacks of saturable reactors.

hysteresis or eddy currents, the top of the curve of Fig. 6 should be perfectly flat with a current of zero. Thus the deviations from flatness inust be due to one of those nonideal properties mentioned above. Because these deviations are quite obvious in the measurements, and because they are expected to have important effects upon interruption, their source has been investigated in considerable detail.

The differences between the effects of finite permeability, hysteresis, and eddy currents can be most readily demonstrated if their separate effects are examined in a situation such as that shown in 


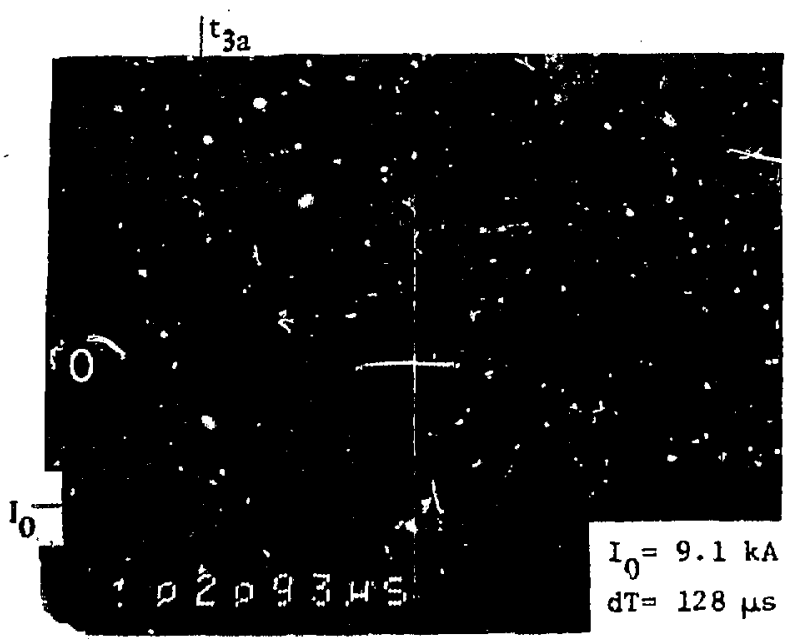

Fig. 18.

Interrupter current with $i_{\mathrm{cp}}$ very low.

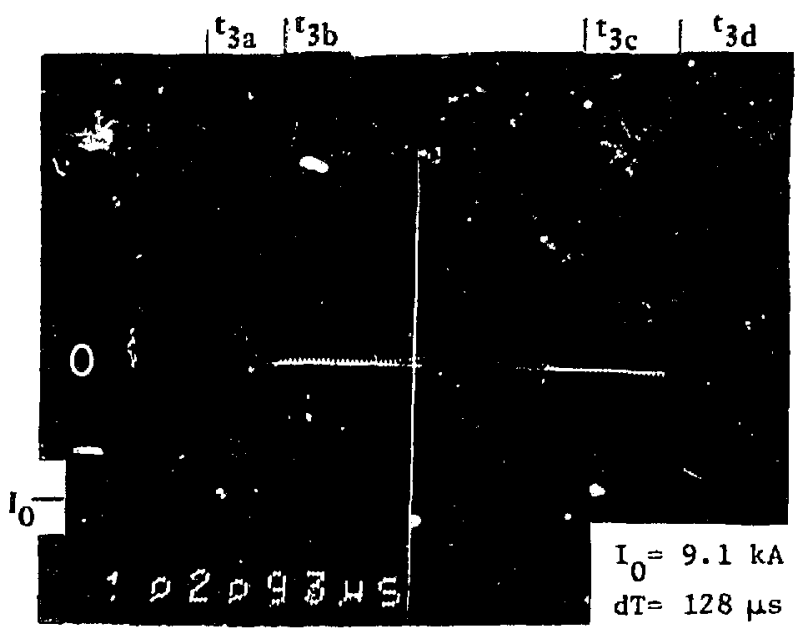

Fig. 19.

Interrupter current with $I_{\mathrm{cp}}$ very high.

Fig. 19, i.e., when the counterpulse is much too large.

If finite permeability is considered alone, the B-H curve of the core can be illustrated by Fig. 20; consequently, the expected counterpulse curve looks like Fig. 21, where the slopes of the two "flat" parts are of the opposite sign but the same magnitude, and the curve is symmetrical about its peak.
If hysteresis is considered alone, the B-H curve loc's like Fig. 22 and the expected counternulse curve locks like. Fig. 23.

A combination of finite permeability and hysieresis will give a curve as in Fig. 24, where the slopes of the "flat" parts still have opposite signs and the seme magnitude, but where the symmetry around the peak has been lost.

Eddy current effects cannot be illustrated with a B-H curve because currents induced in the iron core must be considered. Before the counterpulse, Io creates a saturated flux in the iron. As $\mathbb{I}$ is decreased by the counterpulse, the core eventually comes out of saturation and a flux change occurs in the iron. This generates an emf in each lamination and a resulting eddy current, $I^{\prime}$, proportional to the conductivity of the iron, in each lamination. Figure 25 illustrates this current. Now consider the iron as a perfect magnetic material characterized by an ideal B-H curve; the total current linking the iron must be zero whenever it is unsaturated. Thus, during this period the interrupter current must be reversed slightly to cancel I' and keep the total linked current at zero. Because of the resistance of the lamination, I' will not persist but will die out in such a way as to produce a counterpulse current curve of reversed slope like that shown in Fig. 6. It can be shown that whenever eddy current effects dominate, the slope of the flat parts of the curve will have a constant negative slope.

The magnitude of these eddy current effects can be emphasized by using thick laminations; the effect of finite inductance by using air gaps in the iron; and the effect of hysteresis by using a "hard" iron with a large coercive force. Figures 26-28 illustrate cases where these three effects have been separated fairly well from each other. These measurements were made under identical conditions on almost identical cores. Figure 26 shows a measurement in good agreement with the treatment of eddy currents given above. For this test the standard gapless core was wound of 4 -mil-thick magnetic material. In contrast, Fig. 27 shows agreement with Fig. 23, i.e., an almost pure hysteresis effect. For this test the lamination was only $2 \mathrm{mil}$ thick and should, therefore, show only one-fourth of the eddy current effect of the 4-mil strip. Finally, Fig. 28 shows a combination of hysteresis and finite 


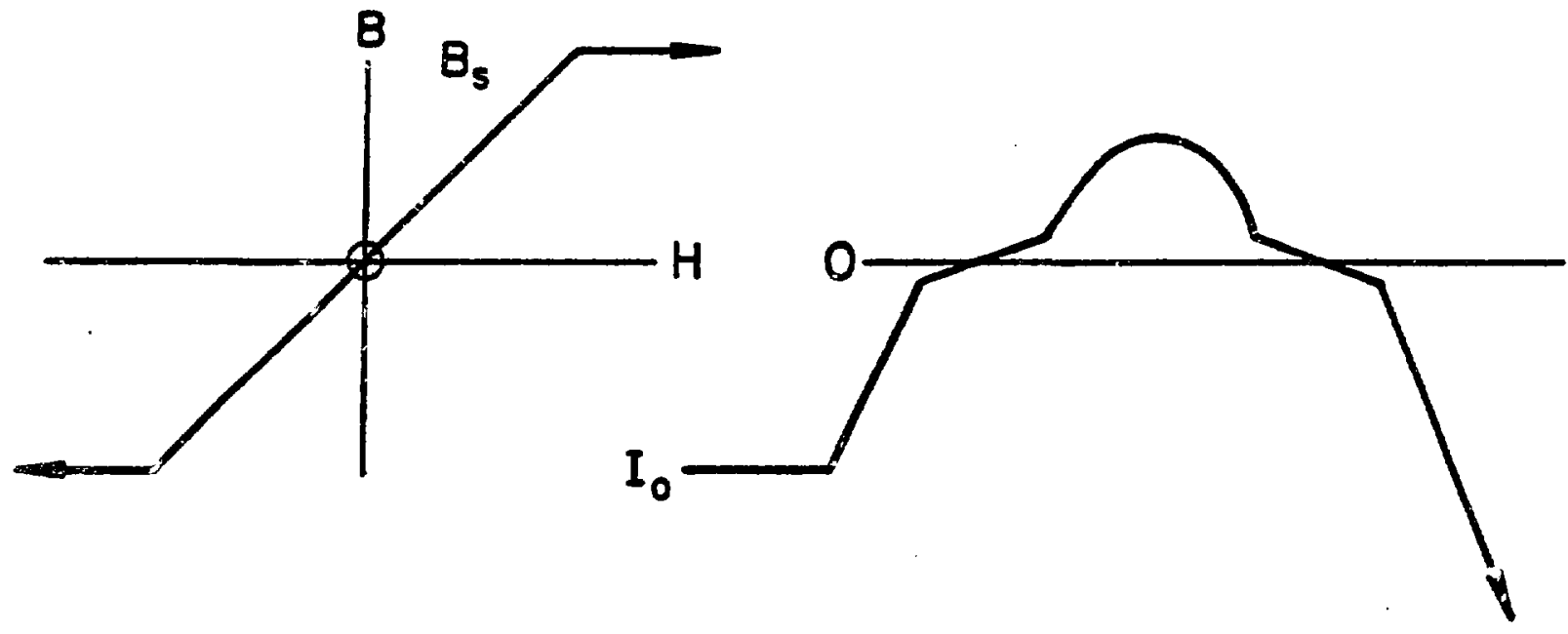

Fig. 20.

$B / H$ curve showing saturation and finite permeability.

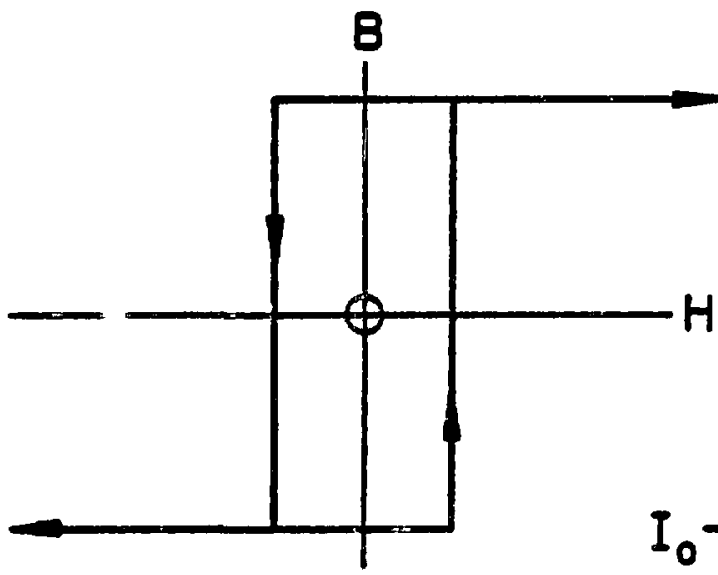

Fig. 22.

$B / H$ curve showing saturation and hysteresis.
Fig. 21.

Hypothetical interrioter current for $B / H$ curve of Fig. 20.
Fig. 23.

Hypothetical interrupter current for $B / H$ curve of Fig. 22. 


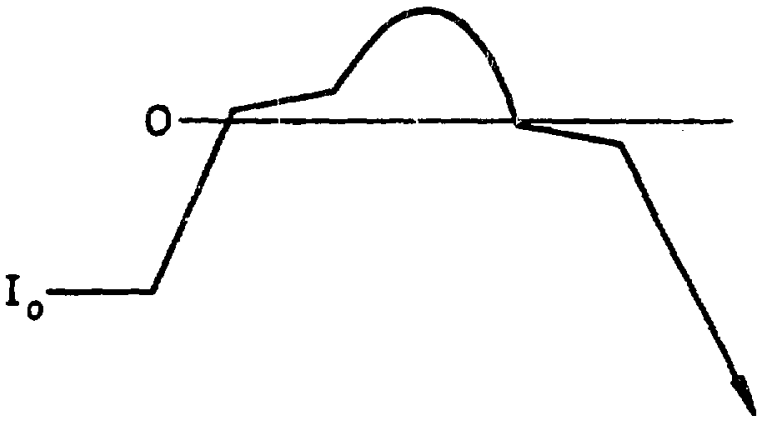

Fig. 24.

Hypothetical interrupter current showing finite permeability and hysteresis.

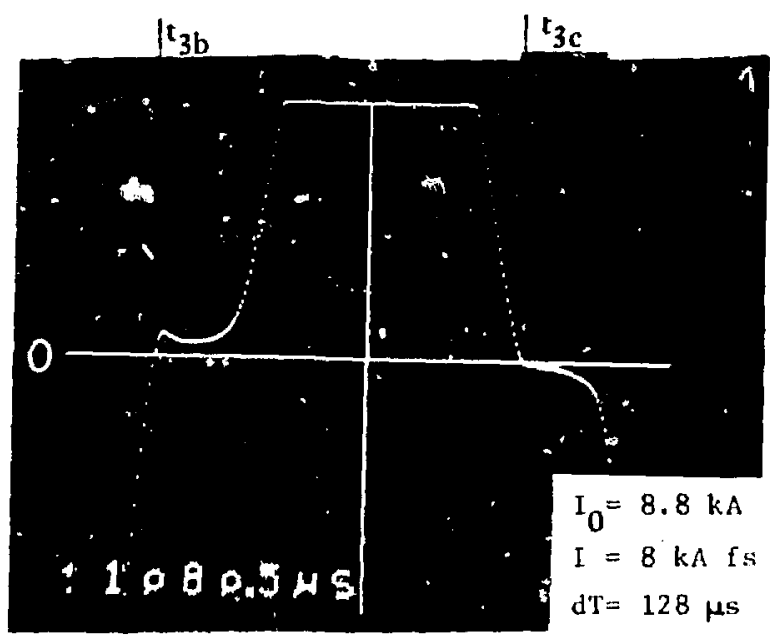

Fig. 26.

Interrupter current showing eddy currents.

perneability. For this test the core was wound with 1-mil strip, the thinnest available. The properties of such a thin strip, for example, its permeability, suffer because of the difficulty of rolling it so thin.

The distortion illustrated in the above paragraphs can also be controlled by external manipulations. If air gaps are purposely introduced into the cores, for example, results, as shown in Fig. 29, typical of finite permeability are readily achieved. The vertical scales of Figs. 27-29 are all the same. In a similar way, extremely large eddy current effects can be introduced by threading a secondary winding through the cores and connecting it to a resistor. By this means the negative slope of Figs. 6 and 26 can

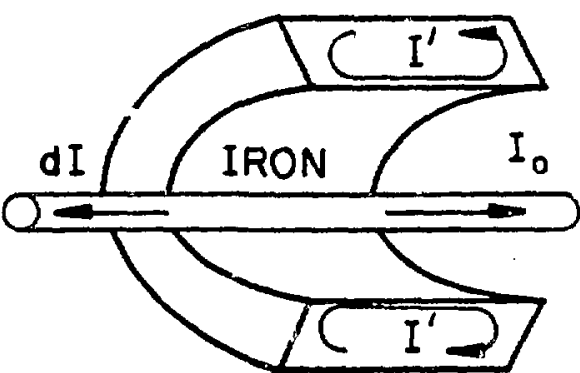

Fig. 25.

Eddy currents in iron laminations.

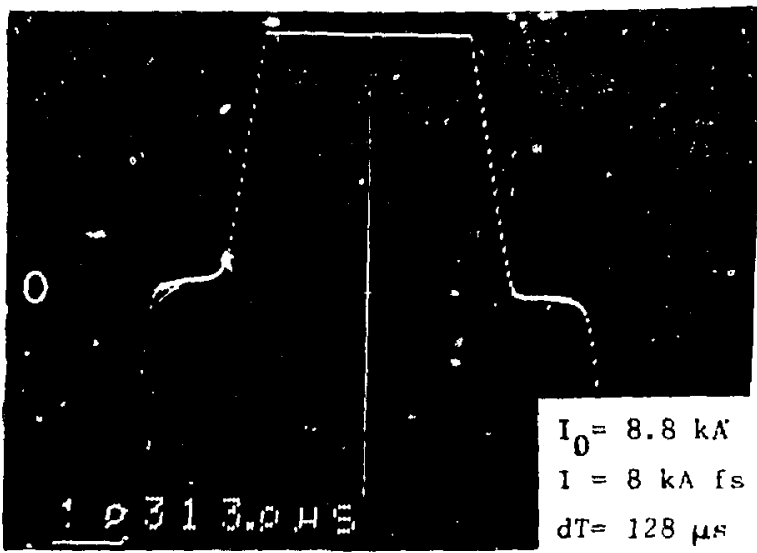

Fig. 27.

Interrupter current showing hysteresis.

be readily increased to any desired value. Suffice it to say that by using either the natural propert.es of different cores or introducing external variations, the shape of the counter pulse can be controlls $\mathrm{i}$ over wide limits. Important effects upon $P$ would be expected to arise from this shape.

Measurements of the dependence of $P$ on (1) the flux rating of the core and (2) the details ef the inductor used are now presented. The discussions begin with the standard $0.08-\mathrm{Wb}$ inductor composed of ten cores. Measurements were made of $P\left(\mathrm{I}_{0}, \mathrm{I}_{\mathrm{cp}}\right)$, and Fig. 30, a graph of the results, was plotted. There are three distinct regions in this plot; Region I where interruption is successsul and no "blip" occurs in the current, race, Region II where interruption is successful and a blip is seen, and Region III where 


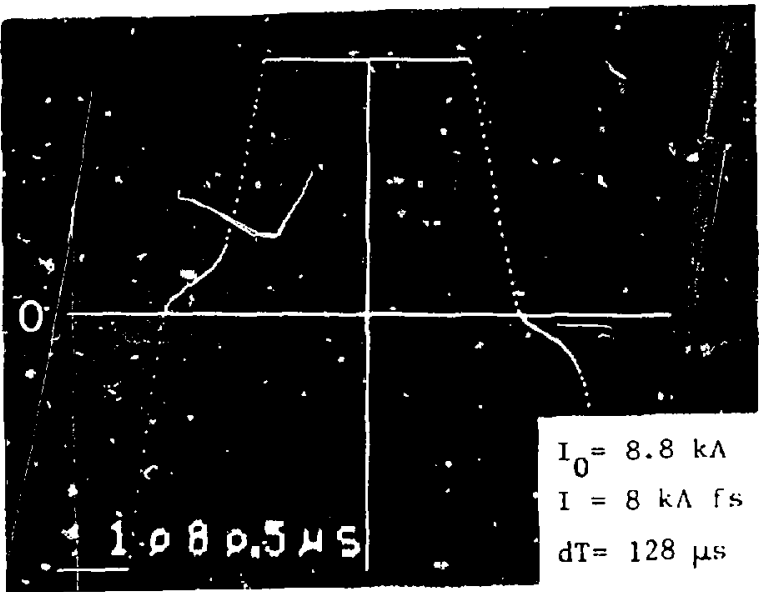

Fig. 28.

Interrupter current showing hysteresis and finite permeability.

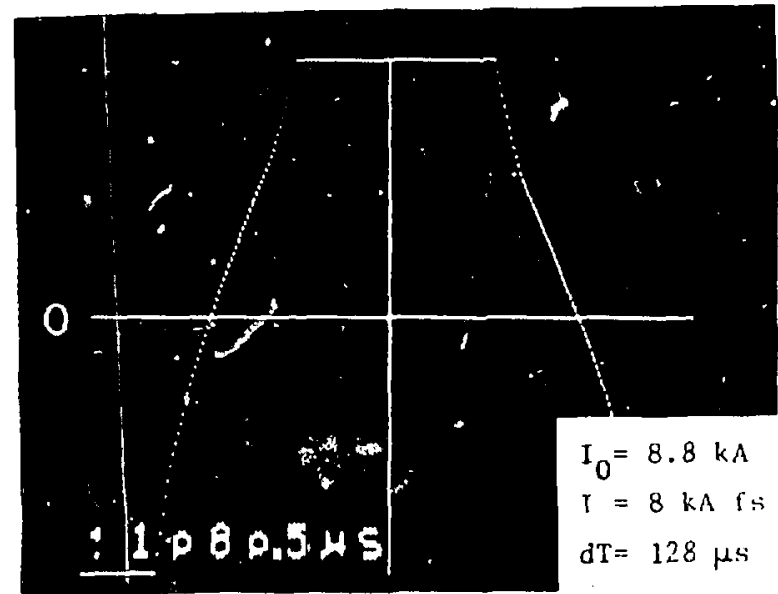

Fig. 29.

Interrupter current showing the effect of air gaps.

interruption fails. The upper boundary of Region I corresponds to the value of $I_{c p}$ at which the saturable reactor is just driven completely through its unsaturated region. This upper boundary can be raised or lowered by using more or fewer cores; however, the effect on $I_{m}$ of changing the number of co'es is insignificant. Thus, the major beneficial effect of using cores is not to increase $I_{m}$ but to extend the range of $I_{c p}$ for a successful interruption. It was also seen that for these tests interruptions made at high currents rarely occur at the second zero. Thus,

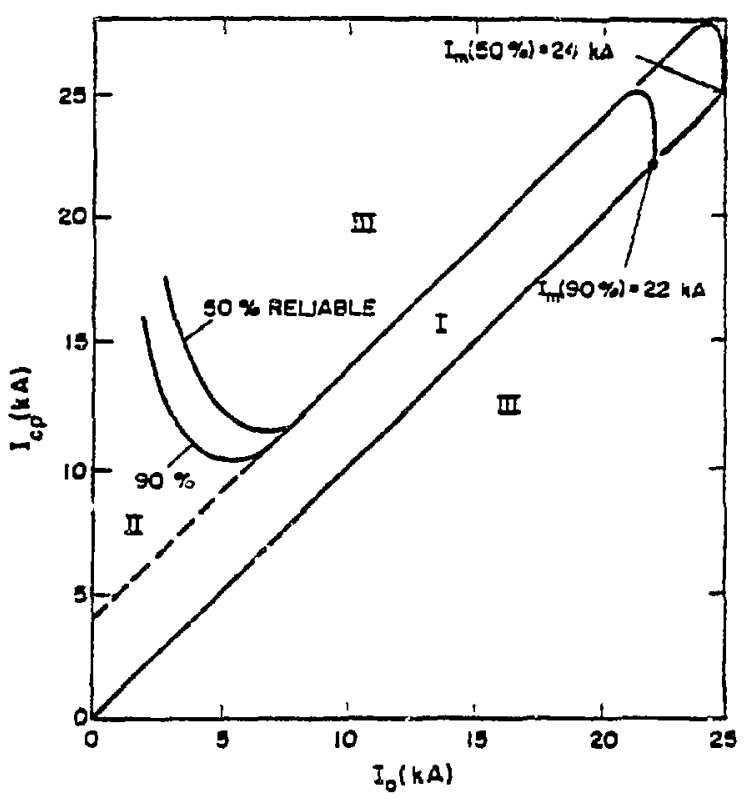

Fig. 30.

Probability of interruption $v s I_{0}$ and $I_{\mathrm{cp}}$ when saturable reactors are used.

it is reasonable that the exact shape of the counterpulse is not important but only the length of time that it holds the interrupter current and voltage near zero. If this time $\delta \mathrm{t}$ is greater than, roughly, 20 $\mu \mathrm{s}$, and if $I_{o}<I_{m}$, the arc interrupts successfully.

Next, $P$ was obtained for six different inductors, all with the same flux value. They were composed of the gapless 1-, 2-, and 4-mil cores discussed above and of the split 4-mil cores, each with spacers of thicknesses 1, 5, and 35 mils in each gap. The countercurrent pulses were quite different in shape for these six cases. Figure 31 , for example, shows the counterpulse current for the split core with two 35mil spacers. It should be compared with Fig. 5 for the standard reactor. In spite of these large differences, $P$ showed almost no dependence on the shape of the counterpulse. Our conclusion from these measurements is that $P$ is determined almost solely by the behavior of the interrupter and that the reactor parameters are quite unimportant as long as $\delta \mathrm{t} \geq 20 \mu \mathrm{s}$. 


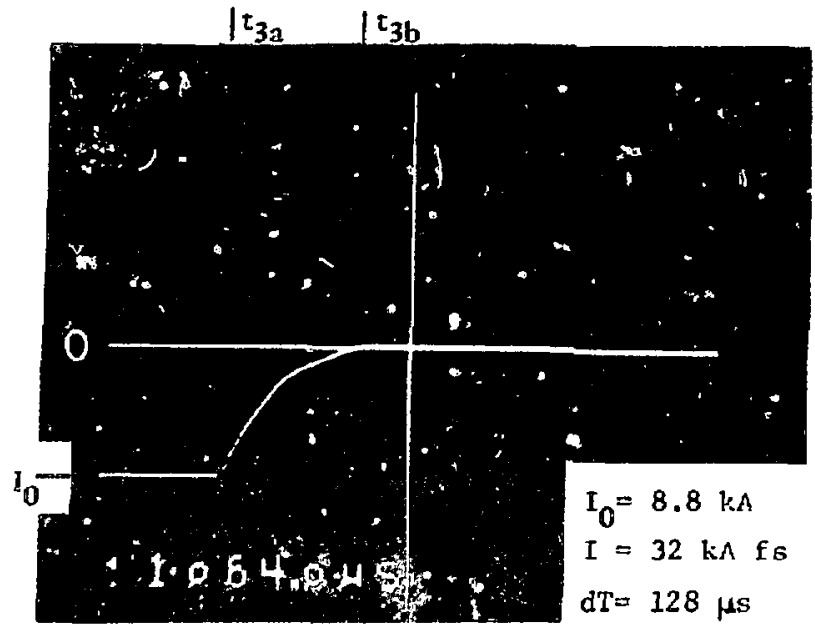

Fig. 31.

Interrupter current with gapped saturable reactor.

\section{F. The Smubber Circuit}

Measurements have been made of $P$ with anc without the snubber circuit and with various value! for $R$. Details of the current and voltage traces de pend upon these parameters and upon whether in. terruption occurs at a first or second zero.

As an illustration of the effects seen, Figs. 32, 33. and 34 show, respectively, the voltage across the in. terrupter when the interrupter was not opened, when it was opened but no snubber circuit was used, and when it was opened and the standard snubber was used.

Figure 32 shows only an inductive voltage pulse generated by the fast-rising counterpulse current For Figs. 33 and 34, the arc was successfully interrupted at the first current zero which occurred on the traces at the vertical fiducial mark. The snubber attenuates the sudden jump in voltage which occurs exactly at interruption time. The currents observed during the voltage traces of Figs. 33 and 34 are very similar. Figure 35 shows an example where, after its first zero, the current reaches a peak of about $100 \mathrm{~A}$ and then decays exponentially 'with a time constant of $20 \mu \mathrm{s}$ or so. This current presumably represents the collection of the plasma residue which was present shortly before interruption when the arc current was much larger, i.e., 9 kA.

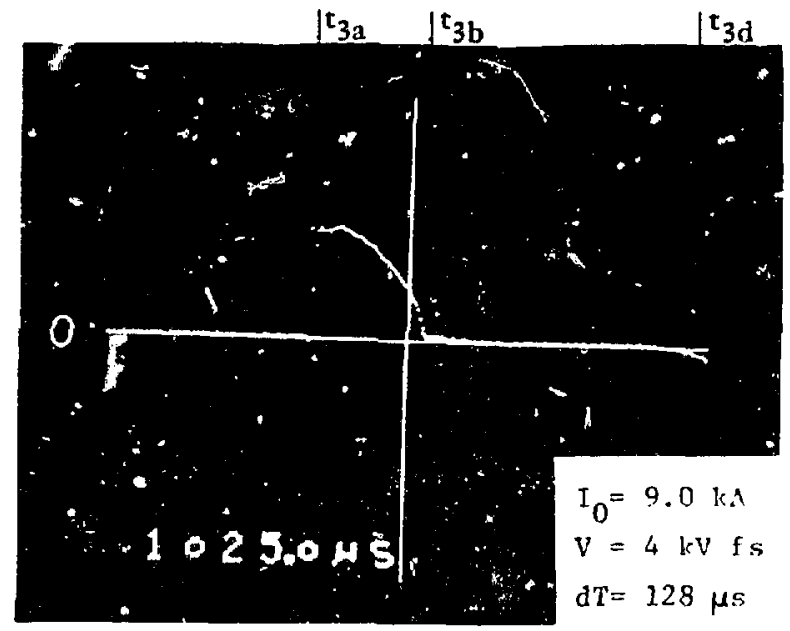

Fig. 32.

Interrupter voltage when interrupter is not opened.

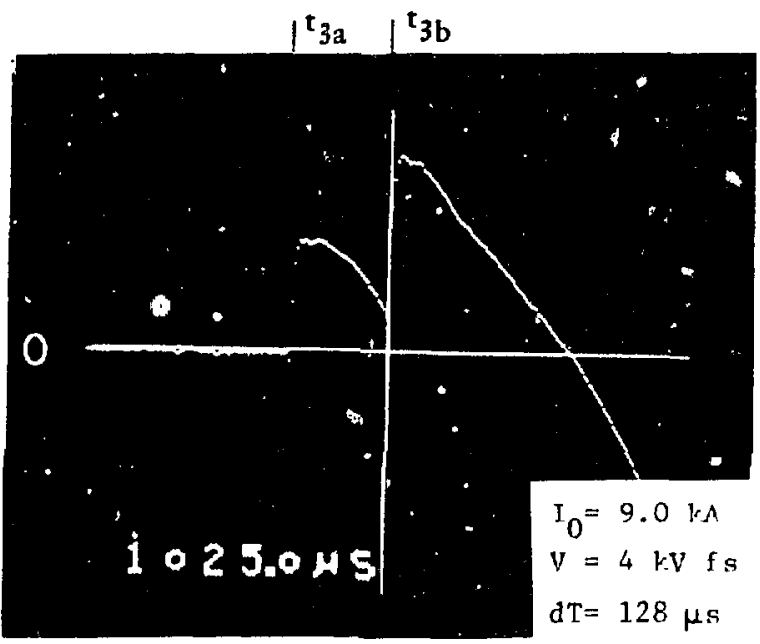

Fig. 33.

Interrupter voltage with no snubber; first current-zero.

In the case of interruption at the second zero, Figs. 36 and 37 show the corresponding voltage traces with no snubber and with the usual one. Again the snubber is effective in eliminating the initial rapid rise in voltage after interruption at $t_{s c}$. In this case the sudden change is downward, and a period of time can be noted between the first zero at 


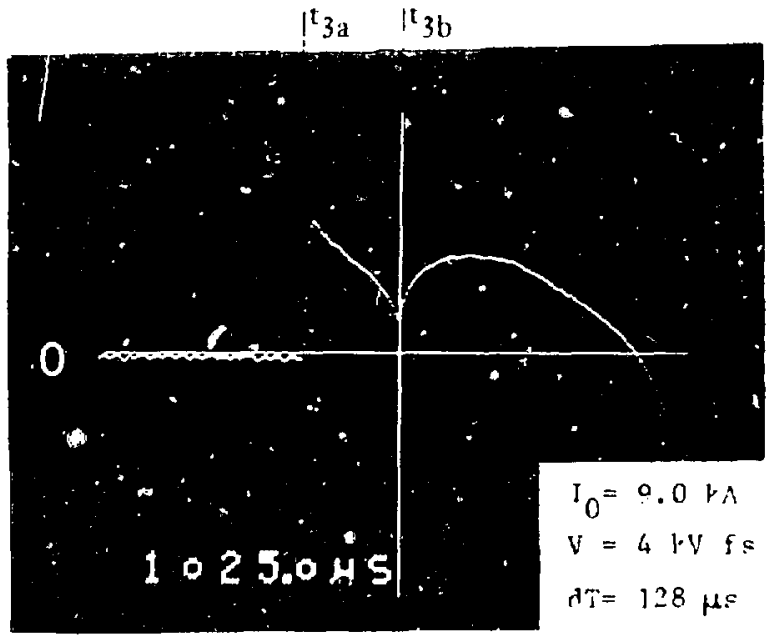

Fig. 34.

Interrupter voltage with snubber; first currentzero.

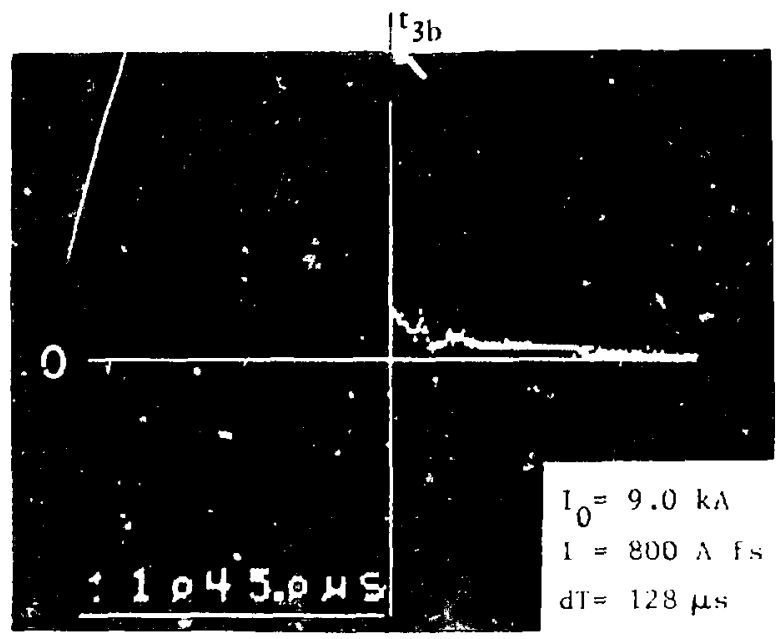

Fig. 35.

Interrupter current near current zeroes.

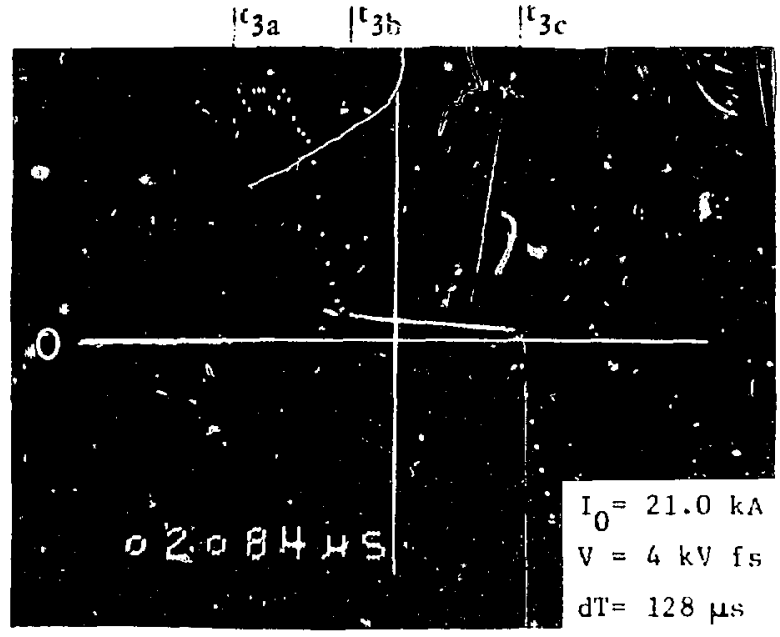

Fig. 36.

Interrupter voltage with no snubier; second current-zero.

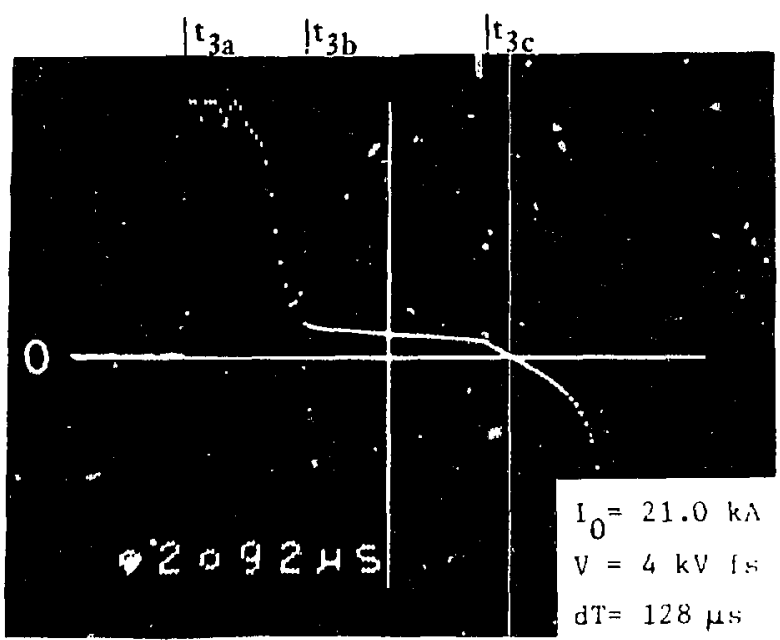

Fig. 37.

Interrupter voltage with snubber; second current-zero. $t_{9 b}$ and the second zero at $t_{3 c}$ during which a lowcurrent $150-\mathrm{V}$ arc burns. A trace of the current observed during a second current zero interruption is shown in Fig. 38. The reversed-slope trace is typical of a saturable reactor dominated by eddy current distortions.

The details of operation of the saturable reactor have more effect on a second-zero interruption than a first. As Figs. 37 and 38 show, the arc current after the first zero flows in a reversed direction. The interrupter voltage adjusts itself to maintain this current. If a standard reactor is used, the current then drops linearly to zero at which time interruption occurs. The interrupter voltage then follows the dictates of $\mathrm{C}_{2}$ and the snubber circuit. If a different reactor had been used, the current trace, Fig. 38, would have been quite different and in most cases, $\mathbf{P}$ would have been lower. 


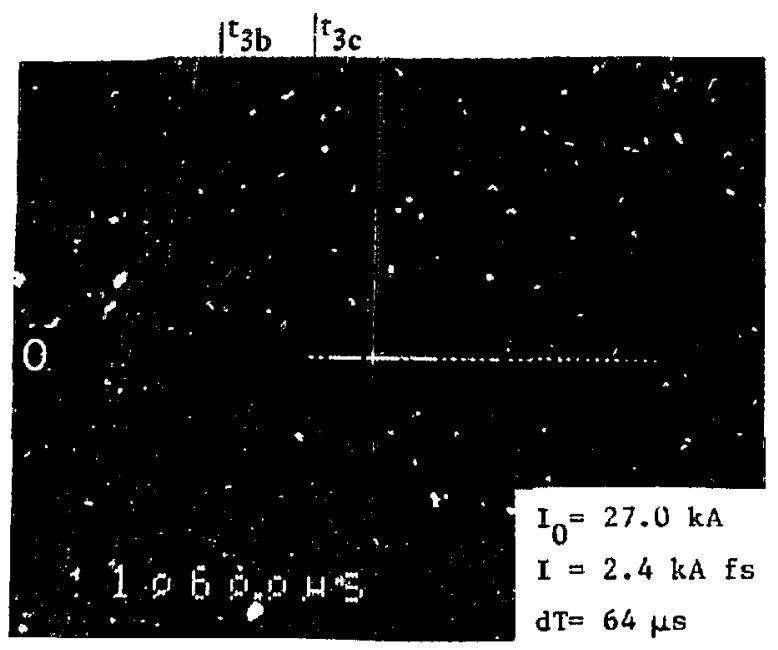

Fig. 38.

Interrupter current near curront zeroes.

If $R$ is set equal to zero, the voltage and current curves are very similar to the above, except that a strong, weakly damped oscillation occurs in the current curve which causes the current to pass rapidly through zero perhaps three or more times during the flat-top time. Curves with $R=0$ and $R$ $=2.3 \Omega$ are shown in Figs. 39 and 40 to illustrate this effect. The interrupter was not opened, thus the counterpulse circuit oscillated through three major cycles on these traces.

Our major finding is that through all of these variations of the snubber circuit, $P$, the probability of interruption is unchanged.

\section{RESULTS FOR DIFFERENT INTTERRUPTERS}

At this point in the investigations all of the commercially available interrupters were tested under standard conditions to determine $P\left(\mathrm{I}_{0}, \mathrm{I}_{c p}\right)$. Tests were also conducted occasionally under nonstandard conditions. From the results, four generalizations and two useful numbers were obtained. The generalizations are:

(1) $P$ does not depend strongly on $I_{c p}$ as long as $I_{c p}$ is large enough to achieve a current zero and the saturable reactor is large enough to suppress a blip.

(2) $P$ does not depend strongly upon $I_{0}$ until the arc voltage becomes noisy. $\mathbb{P}$ then drops rapidly with further increases in $\mathbf{I}_{\mathbf{0}}$.

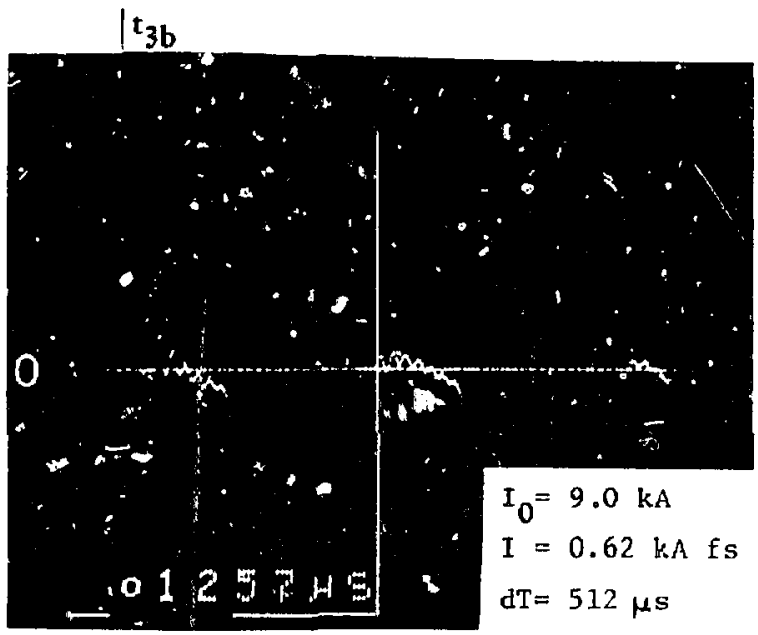

F'.g. 39.

Interrupter current showing ringing of snubber circuit.

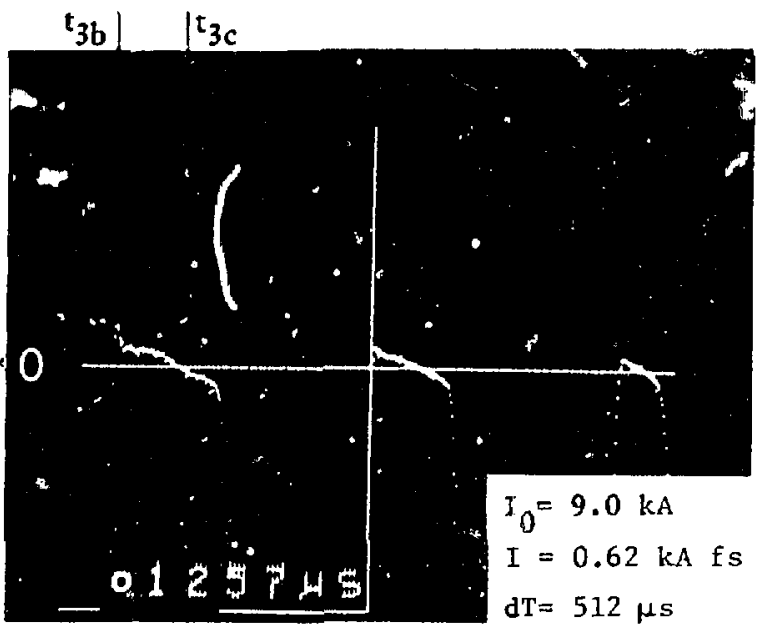

Fig. 40.

Interrupter current without ringing of snubber circuit.

(3) $I_{m}$, this upper limit to $I_{0}$, varies roughly linearly with the diameter of the arcing electrodes.

(4) $I_{m}$ is independent of circuit elements and operational parameters, i.e., saturahle reactor, snubber circuit, opening speed and gap, over a wide range of variation.

(5) There is no significant variation of $I_{m}$ with interrupters from different manufacturers. 
The useful numbers are $I_{m}(50 \%)$, and $I_{m}(90 \%)$, the currents which can re interrupted with a $50 \%$ and $90 \%$ reliability for each different interrupter (see Table I).

Figure 41 shows the linear manner in which $I_{m}$ $(50 \%)$ and $I_{m}(90 \%)$ vary with electrode diameter.

\section{EFFECT OF MAGNETIC FIELD}

There have been several reports ${ }^{3}$ of an improvement in the interruption of $60-\mathrm{Hz}$ arcs through the use of axial magnetic fields. To investigate this effect for dc arcs, a 39-turn solenoid of 4-0 cable was wound which compietely encloses the vacuum bottle, as shown in Fig. 42. The snubber is on the righr. The coil was wound so that its impedance, $\sim 10 \mathrm{~m} \Omega$, would match a $10-\mathrm{V}, 1-\mathrm{kA}$ power supply which was available. With this combination, a maximum field of about $0.8 \mathrm{kG}$ can be generated and maintained for several seconds without overheating the coil.

So far six interrupters have been tested with an axial field, tive with this externally applied field and one special interrupter with an internally generated

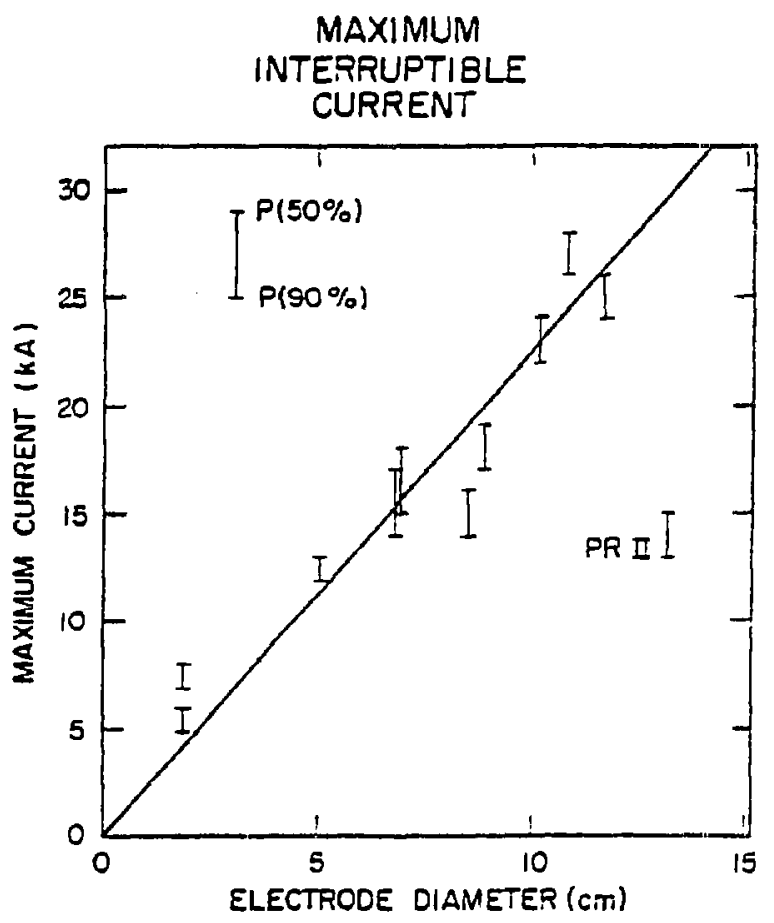

Fig. 41.

Maximum interruptible current for different interrupters.

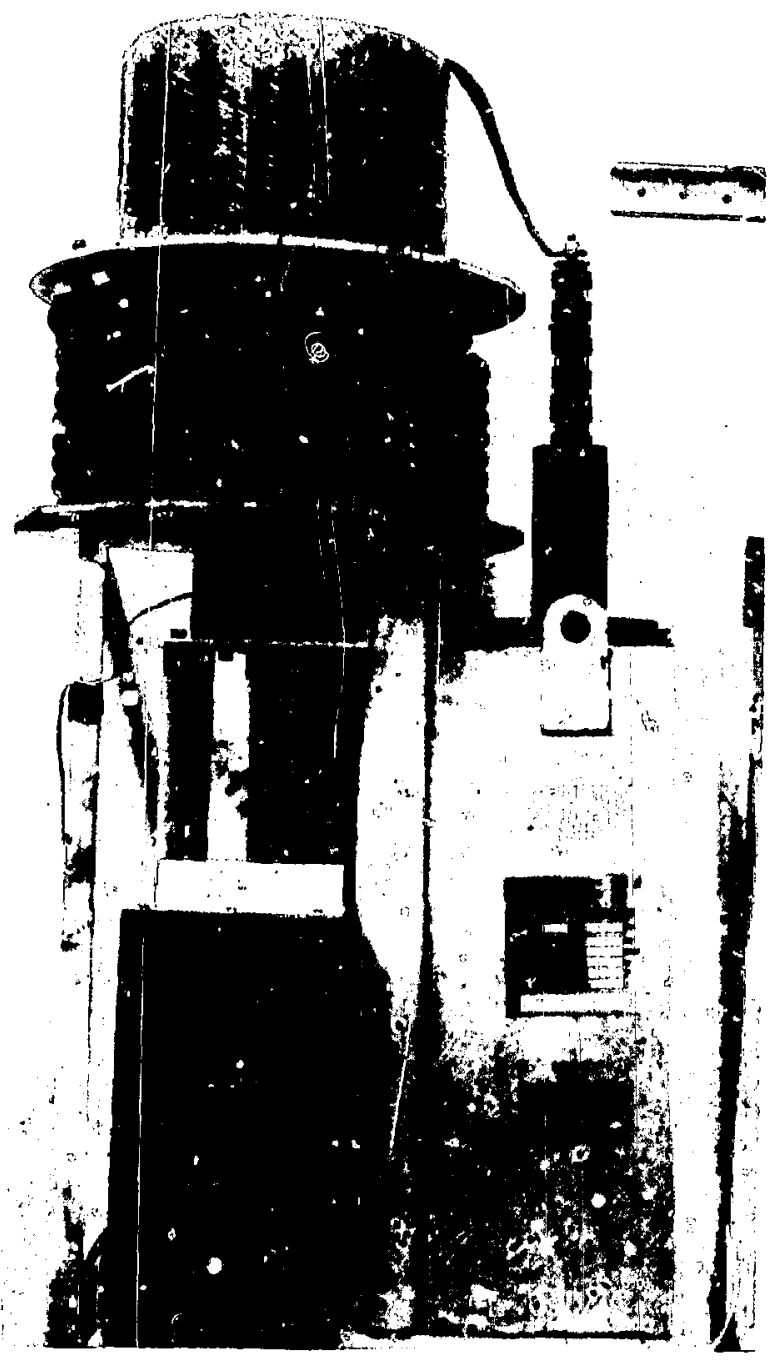

Fig. 42.

Arrangement of field coil around interrupter.

field. The data from these interrupters are shown in Table I. This latter interrupter, called Prototype 1 in Table I, was built by Westinghouse Electric Corp. Prototype 2, also specially built by Westinghouse, has no internal field structure, but contairis electrodes of a novel configuration. Its performance is shown in Fig. 43. The arc voltage is plotted vs magnetic field with $I_{0}$ as a parameter. The open points indicate successful in'erruptions and the solid points, restrikes. The dotted line roughly indicates the boundary between successful interrup. tions and failures. The restrikes occur at progressively lower voltages as the experimental 
EFFEI:T OF AXIAL FIELOS

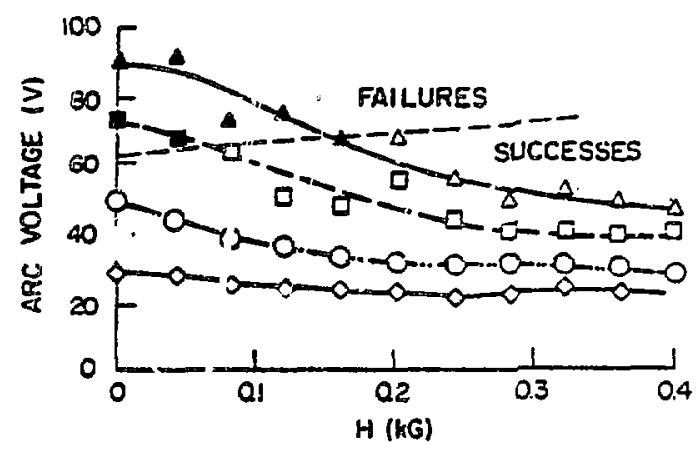

Fig. 43.

Arc vol age versus axial field and arc current.

conditions shift farther som this line. The rating of this interrupter with no krinl field is $13.18 \mathrm{kA}$; with a $0.4-\mathrm{kG}$ fiel. $\mathrm{d}$ the rating is above $30 \mathrm{kA}$. At higher fields, it appears that the rating would continue to improve.

The arc voltage is plotted vs field because of the high sensitivity of the arc voltage to the field. Figures 44 and 45 show the arc voltage vs time for, respectively, an interruption with no field and with a field of about $0.5 \mathrm{kG}$, both carrying the same current. The vertical fiducial is the time when arc voltage shown in Fig. 43 was measured. Clearly, the magnetic field has not only reduced the arc voltage but has also eliminated the noise and reduced the slope of the voltage curve. The same correlation between appearance of noise and failure to interrupt (discussed earlier) appears here. The major effect of the field is therefore identified as a suppression of the anode hot spot. This phenomenon has been directly observed by others."

Two unesplained phenomena are the different magnitudes and the lack of correlation of the field's effect on $I_{m}$ and on the arc voltage. As shown in Table $I$, the improvement in $I_{m}$ varies from $10 \%$ to over $100 \%$ in a regular way, while the reduction in arc voltage varies from 10 to $50 \%$ randomly.

The reductic arc voltage and the presumed absence of an spot when an axial field is used increase $I_{m}$ and are expected to reduce electrode erosion. The magnetic field can be generated in three

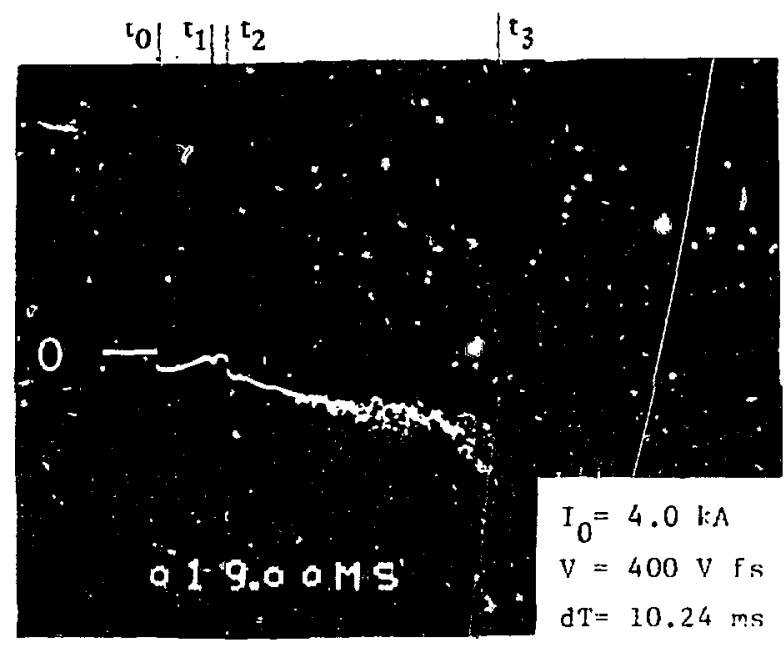

Fig. 44.

Interrupter voltage without axial field.

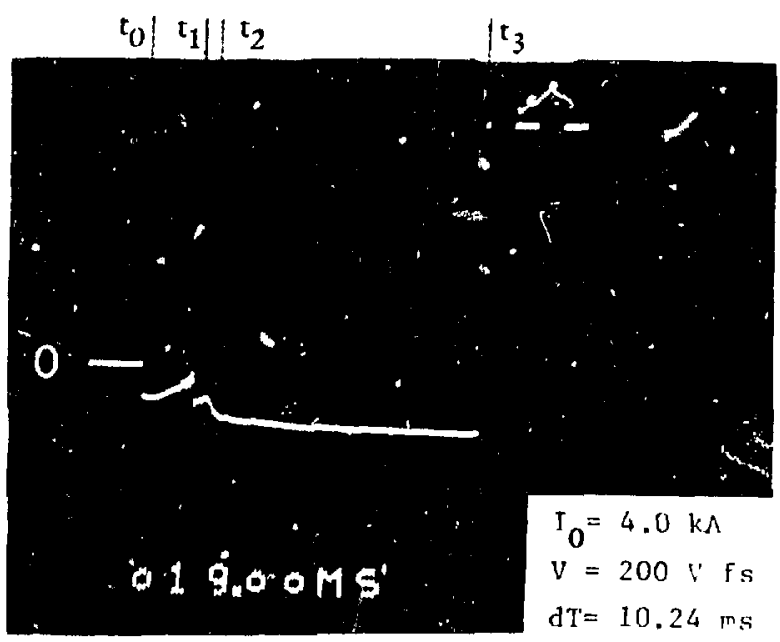

Fig. 45.

Interrupter voltage with axial field.

ways: by an external coil fed by an independent power supply, by an internal coil as accomplished with Prototype 1 , or with an external coil fed by $L_{1}$. The versatility and relative ease of these methods, as well as the large improvements in $I_{m}$ and the assumed reduction in erosion, make the use of axial fields very attractive. This technique presents an obvious means of extending interrupter performance in almost any conceivable application. Other 
interrupters are to be tested at still larger fields to explore these effects further.

\section{LIFI TIISSTS}

\section{A. Mechanical}

Since the number of operations demanded from vacuum interrupters can be many thousands or, for some future uses, millions, we have begun to assess the possible lifetime of standard components. $\mathrm{Ob}$ viously, testing systems in a realistic way for these numbers of cycles can be a time-consuming operation. Synthetic tests of varicus kinds for different vulnerable pa:ts of the system have, therefore, been devised.

The first vulnerable component was thought to be the bellows of the interrupter. Accordingly, interrupters have been opened and closed repeatedly on a standard actuator in a 10-s cycle. Three 7-in. interrupters were cycled in this way while carrying no current. After each operation a high voltage was applied across the open interrupter. A significant current would indicate a vacuum leak. $A$ bad leak could also be detected by a change in the $25-\mathrm{lb}$ force attributable to the vacium helping to hold the electrodes closed.

The first interrupter tested was cycled to 74000 operations. There was no problem with the interrupter during the test. The rest of the actuating system, however, gave many problems. Among these were (1) at 10000 cycles a nut holding the moving stem locsened, (2) at 15000 cycles the high-voltage test circuit burned out, (3) at 28000 cycles a microswitch in the actuator failed, (4) at $29000 \mathrm{cy}$ cles the latching solenoid in the actuator burned out, and (5) at 36000 cycles the connectors to a microswitch sheared off and an aluminum link, which was showing serious wear, wes replaced with a steel link. These problems were not considered serious because the actuator had not been specifically designed for life testing.

The sec ind interrupter was tested successfully to 10400 cycles. A spring and another microswitch failed during these shots. The third interrupter was tested successfully for 10900 cycles, and no new problems were encountered.

At one time an interest arose in cooling electrodes by passing $\ell \mathrm{N}_{2}$ to them through the stems. Doubts were raised about the ability of the in: 'rrupters to withstand the thermal stresses associated with this treatment, so a standard 7-in. interrupter was totally immersed in $\ddot{z} \mathrm{~N}_{\mathrm{z}}$ to see what would happen. Initially this was done very gingerly, scispending the bottle in the cool vapor over the liquid for several hours before immersion, etc. The submergence was so uneventful, however, that the final tests were made by plunging a warm interrupter directly intc $\ell \mathrm{N}_{2}$. No failures of any sort were observed after repezted tests of this kind. Mechanically, these interrrupters aire very pesistant to thermal stress damage.

\section{B. Electrical}

Erosion of the electrodes by the electric arc is now expected to be the serious life-limiting process. Estimates made from experience with $60-\mathrm{Hz}$ applications indicate that a 10000 -operation life expectancy is an optimistic guess. The normal testing procedures for $P\left(I_{0}, I_{c p}\right)$ give some information about this kind of erosion limit, but there are complications. In determining $P$, there are at least four types of interruptions: (1) successful ones, (2) unsuccessful ones because of a counterpulse which is too small, (3) unsuccessful ones where the arc restrikes immediately at a low voltage, ard (4) unsuccessful ones where the restrike is delayed to a high voltage and the restrike current is very large. In the normal operation of an interrupter, however, nost interruptions will be type 1 , i.e., successful ones. In the determinations of $P$, the mixture of interruption types that uccurs gives a larger erosion than would occur if they had all been successful.

Because of this, a special erosion test was made on fresh units in which erosion from successful interruptions could be separated from that due to other types. The erosion measurements were based on observations of the positions of marker points on each electrode's stem. As the electrodes eroded these marker points moved closer together. Although the measurements were complicated by thermal expansion and by stretching of the stem, separate erosion rates for successful and unsuccessful interruptions were obtained at $20 \mathrm{kA}$. In one kind of interrupter, these rates were $0.015 \mathrm{~mm} / \mathrm{shor}$ and $0.08 \mathrm{~mm} / \mathrm{shot}$, respectively. Thus, as few as 
several hundred successful interruptions would exceed the allowable erosion of this interrupter and end its life. In another kind of inter upter, ihese rates were at least an order of magnitude lc wer, allowing a useful life of several thousands of operations. These two types of interrupters contain electrodes made of very different materials. This probebly accounts for the markedly different erosion races.

\section{FUTURE WORK}

\section{A. Refine Ratings of Existing Interrupters}

The easy measurements on vacuum interrupters have now been made. We want to make fordr more important but difficult and time-consuming sets of measurements on conventional interrupters.

1. Determine the dependences of $\mathbf{P}$ on recovery voltage and its time derivative. Preliminary measurements indicate that these dependencies are weak.

2. Determine the effect on $\mathbf{P}$ of the heating of the interrupter caused by an extended passage of current, e.g., a current ramp to $20 \mathrm{kA}$ for $5 \mathrm{~min}$. No substantial experimental information on this effect exists, but it is believed to be small for theoretical reasons.

3. Determine the erosion rate of interrupters used with axial fieids.

4. Determine $I_{m}$ when the current is low enough so that $\mathbf{P}=99 \%$ or more. To make this determination, the number of interruptions must be about 1000 for a $99 \%$ determination and 10000 for $99.9 \%$.

During this very large number of operations we can evaluate simultaneously $I_{m}(99 \%), I_{m}(99.9 \%)$, erosion, and other related lifetime concerns. A knowledge of $I_{m}(99 \%)$ and $I_{m}(99.9 \%)$ is particularly important for switch applications where the reliability of operations must be very high. Conventionai wisdom has it that vacuum interrupters cannot achieve $\mathbf{P}$ values as high as $99 \%$ due to a fundamental limitation. If true, a series arrangement of interrupters to improve reliability will te needed.

\section{B. Increasing Ratings of Existing Interrupters}

Conventional 7 -in. int errupters are to be tested to their limits with an impressed axial field. Conventional interrupters ar to be connected in parallel and series combizations to achieve higher ratings. Some of these roultiple-interrupter arrengements will include axial field interrupters.

\section{New Designs of Interrupters}

The design and manufacture of better interrupters, in particular, better axial field interrupters, is to be promoted, and these new devices are to be tested.

\section{Early Counterpulsing}

The feasibility of a new ${ }^{5}$ approach to interruption with early counterpulsing is to be investigated. With this technique, the counterpulse reduces the current through the interrupter to very low values before the interrupter is opened. Very little arcing or erosion should occur. This is the only technique that appears to be able to achieve an erosion rate so low that lifetimes significantly longer than 10000 operations might be anticipated." To permit this new technique to be used, interrupters and actuators of much higher speed must be constructed. The feasibility of this goal is being investigated through interactions with several developers and manufacturers of interrupters and actuators.

\section{INTERRUPTER MEASUREMENTS AND DEVELOPMENT WORK PERFORMED ELSEWHERE}

All tokamaks need large interrupting switches to operate their ohmic heating circuits. It is to be expected, therefore, that work paralleling that

- Similar work is being done at Hughes Research Joratory by W. Knauer. 
described above would be performed wherever tokamaks are being built e.g., Russia, Europe, and Japan. Some of the work in Europe involves vacuum iriterrupters ${ }^{\theta}$ and some air-blast interrupters. The most advanced work on vacuum interrupters appears to have been performed by Toshiba? in Japan for JT-60. They use axial magnetic fields as an essential feature.

\section{ACKNOWLEDGMENTS}

The test circuit used in this work was originally assembled and tested and preliminary component kesting was begun by Clarke Swannack and other Staff Members of CTR-9. The work performed by the author was based upon these cuiy contributions and benefitted greatly from the continued guidance of Clarke Swannack.

\section{REFIRENCES}

1. C. $\mathbb{E}$. Swannack and $\mathbb{K}$. D. Williamson, Jr., "Superconducting Magnetic and Inertial Energy Pulsed Power Systems," Proc. Intern. Conf. on Radiation Test Facilities for the CTR Surface and Materials Program (July 1975), pp. 807-829.

2. H. C. Miller, "Vacuum Arc Anode Phenomena," Document 76NOD-011, US Energy Research and Development Administration report, Coniract EY76-C-04-0656; C. W. Kimblin, "Anode Voltage Drop and Anode Spot Formation in DC Vacuum Arcs," J. Appl. Phys. 40, 1744 (1969).
3. C. W. Kimbli : and R. E. Vorshall, "Intermuption Ability of Vacuum Interrupters Subjected to Axial Magnetic Fields," Proc. Inst. Elect. Eng. 119 1754-8 (1972); T. Ito and T. M. Ohkura, "High Current In- . terruption Phenornena in Vacuum Inter:upters," Mitoubishi Denki Giho A1, 1409 (1967).

4. O. Morimiya, S. Sohma, T. Sugawara, and $\mathbb{H}$. Mizutani, "High Current Vacuum Arce Stabilized by Axial Magnetic Fields, "IEEE Trans. Power App. Syst. PAS-92 1723-32 (1973); C. W. Kimblin and R. E. Voshall, "Vacuum Arcs Subjected to Axial Magnetic Fields," XI Int. Conf. Phen. Ion. Gases 78 (Prague, 1973); W. G. J. Rondeel, "The Confinement of a Vacuum Arc in an Axial Magnetic Field," Proc. VI Int. Sym. Dis. Elec. Insul. Vac. 241-246 (Swansea, 1974).

5. P. Dokopoulos and K. Kriechbaum, "DC Circuit Breakers for $73 \mathrm{kA}, 24 \mathrm{kV}$," Elektratechnische Zeitschrift, A, 97, pp. 499-503, August 1976.

6. P. Dokopoulos and D. Smart, "DC Current Breaking Test on Vacuum Interrupters," Proc. 9th Symp. on Fusion Technology (Pergamon Press, Oyford \& New York, 1976), p. 759.

7. "Poloidal Field Power Supply Using Vacuum Circuit Breakers," submitted to IAEA 2nd Large Tokamak Meeting, Princeton, November 1976. 